Article

\title{
Some Mann-Type Implicit Iteration Methods for Triple Hierarchical Variational Inequalities, Systems Variational Inequalities and Fixed Point Problems
}

\author{
Lu-Chuan Ceng ${ }^{1}$ and Xiaoye Yang ${ }^{2, *}$ \\ 1 Department of Mathematics, Shanghai Normal University, Shanghai 200234, China; zenglc@hotmail.com \\ 2 Department of Mathematics, Ren'ai College, Tianjin University, Tianjin 301636, China \\ * Correspondence: xyyang33@126.com; Tel.: +86-138-2189-9617
}

Received: 31 December 2018; Accepted: 21 February 2019; Published: 26 February 2019

\begin{abstract}
This paper discusses a monotone variational inequality problem with a variational inequality constraint over the common solution set of a general system of variational inequalities (GSVI) and a common fixed point (CFP) of a countable family of nonexpansive mappings and an asymptotically nonexpansive mapping in Hilbert spaces, which is called the triple hierarchical constrained variational inequality (THCVI), and introduces some Mann-type implicit iteration methods for solving it. Norm convergence of the proposed methods of the iteration methods is guaranteed under some suitable assumptions.
\end{abstract}

Keywords: mann-type implicit iteration methods; triple hierarchical constrained variational inequality; general system of variational inequalities; strong convergence; hilbert spaces

\section{Introduction}

Let $C$ be a convex closed nonempty subset of a real Hilbert space $H$ with norm $\|\cdot\|$ and inner product $\langle\cdot, \cdot\rangle$. Let $P_{C}$ be the metric (or nearest point) projection from $H$ onto $C$, that is, for all $x \in H, P_{C} x \in C$ and $\left\|x-P_{C} x\right\|=\inf _{y \in C}\|x-y\|$. Let $T: C \rightarrow C$ be a possible nonlinear mapping. Denote by $\operatorname{Fix}(T)$ the set of fixed points of $T$, i.e., $\operatorname{Fix}(T)=\{x \in C: x=T x\}$. We use the notations $\mathbf{R}, \rightarrow$ and $\rightarrow$ to indicate the set of real numbers, weak convergence and strong convergence, respectively.

A mapping $T: C \rightarrow C$ is said to be asymptotically nonexpansive (see [1]), if there exists a sequence $\left\{\theta_{n}\right\} \subset[0,+\infty)$ with $\lim _{n \rightarrow \infty} \theta_{n}=0$ such that

$$
\left\|T^{n} x-T^{n} y\right\| \leq\left(1+\theta_{n}\right)\|x-y\| \quad \forall n \geq 0, x, y \in C .
$$

In particular, $T$ is said to be nonexpansive if $\|T x-T y\| \leq\|x-y\|, \forall x, y \in C$, that is, $\theta \equiv 0$. If $C$ is also a bounded set, then the fixed-point set of $T$ is nonempty, that is $\operatorname{Fix}(T) \neq \varnothing$. Via iterative techniques, fixed points of (asymptotically) nonexpansive mappings have been studied because of their applications in convex optimization problems; see [2-10] and the references therein.

Let $B_{1}, B_{2}: C \rightarrow H$ be two nonlinear single-valued mappings. We consider the following problem of finding $\left(x^{*}, y^{*}\right) \in C \times C$ such that

$$
\begin{cases}\left\langle x-x^{*}, \mu_{1} B_{1} y^{*}+x^{*}-y^{*}\right\rangle \geq 0, & \forall x \in C, \\ \left\langle x-y^{*}, \mu_{2} B_{2} x^{*}+y^{*}-x^{*}\right\rangle \geq 0, & \forall x \in C,\end{cases}
$$

which is called a general system of variational inequalities (GSVI) with real number constants $\mu_{1}$ and $\mu_{2}>0$, which covers as special subcases the problems arising, especially from nonlinear 
complementarity problems, quadratic mathematical programming and other variational problems. The reader is referred to [11-18] and the references therein. Particularly, if both $B_{1}$ and $B_{2}$ are equal to $A$ and $x^{*}=y^{*}$, then problem (1) become the classical variational inequality (VI), that set of solutions is stated by $\operatorname{VI}(C, A)$. Note that, problem (1) can be transformed into a fixed-point problem in the following way.

Lemma 1 ([19]). Let both $x^{*}$ and $y^{*}$ be points in $C .\left(x^{*}, y^{*}\right)$ is a solution of GSVI (1) if and only if $x^{*} \in$ $\operatorname{GSVI}\left(C, B_{1}, B_{2}\right)$, where $\operatorname{GSVI}\left(C, B_{1}, B_{2}\right)$ is the fixed point set of the mapping $G:=P_{C}\left(I-\mu_{1} B_{1}\right) P_{C}(I-$ $\left.\mu_{2} B_{2}\right)$, and $y^{*}=P_{C}\left(I-\mu_{2} B_{2}\right) x^{*}$.

A mapping $A: C \rightarrow H$ is called monotone if

$$
\langle A x-A y, x-y\rangle \geq 0, \quad \forall x, y \in C
$$

It is called $\eta$-strongly monotone if there exists a constant $\eta>0$ such that

$$
\langle A x-A y, x-y\rangle \geq \eta\|x-y\|^{2}, \quad \forall x, y \in C .
$$

Moreover, it is called $\alpha$-inverse-strongly monotone (or $\alpha$-cocoercive), if there exists a constant $\alpha>0$ such that

$$
\langle A x-A y, x-y\rangle \geq \alpha\|A x-A y\|^{2}, \quad \forall x, y \in C .
$$

Obviously, each inverse-strongly monotone mapping is monotone and Lipschitzian, and each strongly monotone and Lipschitzian mapping is inverse-strongly monotone but the converse is not true.

Recently, Cai et al. [20] proposed a new implicit-rule for obtaining a common element of the solution set of GSVI (1) and the fixed point set of an asymptotically nonexpansive mapping $T$, and presented norm convergence of the sequence generated by the proposed rule to an element of $\operatorname{GSVI}\left(C, B_{1}, B_{2}\right) \cap \operatorname{Fix}(T)$, which also solves certain VI.

On the other hand, Iiduka [21] considered a monotone variational inequality linked to a inequality constraint over the set of fixed points of a nonexpansive mapping. Iiduka's problem is a triple mathematical programming in contrast with bilevel mathematical programming problems or hierarchical constrained optimization problems or nonlinear hierarchical problem, it is referred as triple hierarchical constrained optimization problem (THCOP). Since the THCOP is a general variational inequality, we also call it a triple hierarchical variational inequality (THVI). This kind of problems play an important role in nonlinear minimizer problems and nonlinear operator equations; see [22-26] and the references therein.

To begin with, let us recall the variational inequality for a monotone mapping, $A_{1}: H \rightarrow H$, over the fixed point set of a nonexpansive mapping, $T: H \rightarrow H$ :

$$
\text { Find } \begin{aligned}
\bar{x} & \in \operatorname{VI}\left(\operatorname{Fix}(T), A_{1}\right) \\
& :=\left\{\bar{x} \in \operatorname{Fix}(T):\left\langle A_{1} \bar{x}, y-\bar{x}\right\rangle \geq 0 \forall y \in \operatorname{Fix}(T)\right\},
\end{aligned}
$$

where $\operatorname{Fix}(T):=\{x \in H: T x=x\} \neq \varnothing$. Iiduka's THCOP and its algorithm (Algorithm 1) are stated below.

Problem 1. (see [21], Problem 3.1) Assume that

(C1) $T: H \rightarrow H$ is a nonexpansive mapping such that $\operatorname{Fix}(T) \neq \varnothing$;

(C2) $A_{2}: H \rightarrow H$ is $\kappa$-Lipschitz continuous $\eta$-strongly monotone;

(C3) $A_{1}: H \rightarrow H$ is $\zeta$-inverse-strongly monotone;

(C4) $\operatorname{VI}\left(\operatorname{Fix}(T), A_{1}\right) \neq \varnothing$. 
Then the objective is to

$$
\text { Find } \begin{aligned}
x^{*} & \in \operatorname{VI}\left(\operatorname{VI}\left(\operatorname{Fix}(T), A_{1}\right), A_{2}\right) \\
& :=\left\{x^{*} \in \operatorname{VI}\left(\operatorname{Fix}(T), A_{1}\right):\left\langle v-x^{*}, A_{2} x^{*}\right\rangle \geq 0 \forall v \in \operatorname{VI}\left(\operatorname{Fix}(T), A_{1}\right)\right\} .
\end{aligned}
$$

Algorithm 1. (see [21], Algorithm 4.1)

Step 0. Take $\left\{\alpha_{n}\right\}_{n=0}^{\infty},\left\{\delta_{n}\right\}_{n=0}^{\infty} \subset(0, \infty)$, and $\mu>0$, choose $x_{0} \in H$ arbitrarily, and let $n:=0$.

Step 1. Given $x_{n} \in H$, compute $x_{n+1} \in H$ as

$$
\left\{\begin{array}{l}
y_{n}=T\left(x_{n}-\delta_{n} A_{1} x_{n}\right), \\
x_{n+1}=y_{n}-\alpha_{n} \mu A_{2} y_{n} .
\end{array}\right.
$$

Update $n:=n+1$ and go to Step 1 .

The purpose of this paper is to introduce and analyze some Mann-type implicit iteration methods for treating a monotone variational inequality with a inequality constraint over the common solution set of the GSVI (1) for two inverse-strongly monotone mappings and a common fixed point problem (CFPP) of a countable family of nonexpansive mappings and an asymptotically nonexpansive mapping in Hilbert spaces, which is called the triple hierarchical constrained variational inequality (THCVI). Here the Mann-type implicit iteration methods are based on the Mann iteration method, viscosity approximation method, Korpelevich's extragradient method and hybrid steepest-descent method. Under some suitable assumptions, we prove strong convergence of the proposed methods to the unique solution of the THCVI.

\section{Preliminaries}

Now we recall some necessary concepts and facts. A mapping $F: C \rightarrow H$ is named to be $\kappa$-Lipschitzian if there is a real number $\kappa>0$ with

$$
\kappa\|x-y\| \geq\|F(x)-F(y)\|, \quad \forall x, y \in C .
$$

Particularly, if $\kappa \in(0,1)$, then $F$ is said to be contractive. If $\kappa=1$, then $F$ is said to be a nonexpansivity. A mapping $A: H \rightarrow H$ is named to be a strongly positive bounded linear operator if there is a real number $\gamma>0$ with

$$
\langle A x, x\rangle \geq \gamma\|x\|^{2}, \quad \forall x \in H
$$

For a fixed $x \in H$, we know that there is a unique point in $C$, presented by $P_{C} x$, with

$$
\|x-y\| \geq\left\|x-P_{C} x\right\|, \quad \forall y \in C
$$

$P_{C}$ is called a metric projection of $H$ onto $C$.

Lemma 2. There hold the following important relations for metric projection $P_{C}$ :

(i) $\left\langle x-y, P_{C} x-P_{C} y\right\rangle \geq\left\|P_{C} x-P_{C} y\right\|^{2}, \forall x, y \in H$;

(ii) $0 \geq\left\langle x-P_{C} x, y-P_{C} x\right\rangle, \forall x \in H, y \in C$;

(iii) $\|x-y\|^{2}+2\langle x-y, y\rangle=\|x\|^{2}-\|y\|^{2}, \forall x, y \in H$;

(iv) $\|x-y\|^{2} \geq\left\|x-P_{C} x\right\|^{2}+\left\|y-P_{C} x\right\|^{2}, \forall x \in H, y \in C$.

Lemma 3 ([27]). Let $\left\{a_{n}\right\}$ be a sequence of real numbers with the conditions:

$$
a_{n+1} \leq\left(1-\lambda_{n}\right) a_{n}+\lambda_{n} \gamma_{n}, \quad \forall n \geq 0,
$$


where $\left\{\lambda_{n}\right\}$ and $\left\{\gamma_{n}\right\}$ are sequences of real numbers such that (i) $\left\{\lambda_{n}\right\} \subset[0,1]$ and $\sum_{n=0}^{\infty} \lambda_{n}=\infty$, and (ii) $\sum_{n=0}^{\infty}\left|\gamma_{n} \lambda_{n}\right|<\infty$ or $\lim \sup _{n \rightarrow \infty} \gamma_{n} \leq 0$. Then $\lim _{n \rightarrow \infty} a_{n}=0$.

Lemma 4 ([27]). Let $\lambda$ be real number in $(0,1]$. Let $T: C \rightarrow H$ be a nonexpansive nonself mapping. Let $T^{\lambda}: C \rightarrow H$ be a nonself mapping defined by

$$
T^{\lambda} x:=T x-\lambda \mu F(T x), \quad \forall x \in C .
$$

Here $F: H \rightarrow H$ is $\kappa$-Lipschitzian and $\eta$-strongly monotone. So, $T^{\lambda}$ is a contraction if $0<\mu<\frac{2 \eta}{\kappa^{2}}$, i.e.,

$$
\left\|T^{\lambda} x-T^{\lambda} y\right\| \leq(1-\lambda \tau)\|x-y\|, \quad \forall x, y \in C,
$$

where $\tau=1-\sqrt{1-\mu\left(2 \eta-\mu \kappa^{2}\right)} \in(0,1]$.

Lemma 5 ([17]). Let the mapping $A: C \rightarrow H$ be $\alpha$-inverse-strongly nonself monotone. Then, for a given $\lambda \geq 0,\|(I-\lambda A) x-(I-\lambda A) y\|^{2} \leq\|x-y\|^{2}+\lambda(\lambda-2 \alpha)\|A x-A y\|^{2}$. In particular, if $0 \leq \lambda \leq 2 \alpha$, then $I-\lambda A$ is nonexpansive.

Lemma 6 ([17]). Let the mappings $B_{1}, B_{2}: C \rightarrow H$ be $\alpha$-inverse-strongly monotone and $\beta$-inverse-strongly monotone, respectively. Let the mapping $G: C \rightarrow C$ be defined as $G:=P_{C}\left(I-\mu_{1} B_{1}\right) P_{C}\left(I-\mu_{2} B_{2}\right)$. If $0 \leq \mu_{1} \leq 2 \alpha$ and $0 \leq \mu_{2} \leq 2 \beta$, then $G: C \rightarrow C$ is nonexpansive.

Lemma 7 ([28]). Let $H$ be a Hilbert space. We suppose that $C$ is a convex closed nonempty set in $H$, and $T$ : $C \rightarrow C$ is an asymptotically nonexpansive nonself mapping with a nonempty fixed point set, that is, $\operatorname{Fix}(T) \neq \varnothing$. Then $I-T$ is demiclosed at zero, i.e., if $\left\{x_{n}\right\} \subset C$ converges weakly to some $x \in C$, and $\left\{(I-T) x_{n}\right\}$ converges strongly to zero, then $(I-T) x=0$, where $I$ is the identity mapping on $H$.

Lemma 8 ([29]). Let $H$ be a Hilbert space. We suppose that $\left\{x_{n}\right\}$ and $\left\{w_{n}\right\}$ are bounded vector sequences in $H$ and $\left\{\beta_{n}\right\}$ is a real number sequence in $(0,1)$ such that $\lim _{\sup _{n \rightarrow \infty}} \beta_{n} \leq 1$ and $\lim _{n \rightarrow \infty} \inf _{n \rightarrow \infty} \beta_{n}>0$. We also suppose that $x_{n+1}=\beta_{n} x_{n}+\left(1-\beta_{n}\right) w_{n}, \forall n \geq 0$ and

$$
\limsup _{n \rightarrow \infty}\left(\left\|w_{n+1}-w_{n}\right\|-\left\|x_{n+1}-x_{n}\right\|\right) \leq 0
$$

Then $\lim _{n \rightarrow \infty}\left\|w_{n}-x_{n}\right\|=0$.

Let $C$ be a convex closed nonempty set. Let $\left\{S_{n}\right\}_{n=0}^{\infty}$ be a countable family of nonexpansive self mappings defined on $C$, and $\left\{\lambda_{n}\right\}_{n=0}^{\infty}$ be a sequence of real numbers in $[0,1]$. On $C$, we define a self mapping $W_{n}$ :

$$
\left\{\begin{array}{l}
U_{n, n+1}=I, \\
U_{n, n}=\left(1-\lambda_{n}\right) I+\lambda_{n} S_{n} U_{n, n+1}, \\
U_{n, n-1}=\left(1-\lambda_{n-1}\right) I+\lambda_{n-1} S_{n-1} U_{n, n}, \\
\cdots, \\
U_{n, k}=\left(1-\lambda_{k}\right) I+\lambda_{k} S_{k} U_{n, k+1}, \\
U_{n, k-1}=\left(1-\lambda_{k-1}\right) I+\lambda_{k-1} S_{k-1} U_{n, k}, \\
\cdots, \\
U_{n, 1}=\left(1-\lambda_{1}\right) I+\lambda_{1} S_{1} U_{n, 2}, \\
W_{n}=U_{n, 0}=\left(1-\lambda_{0}\right) I+\lambda_{0} S_{0} U_{n, 1} .
\end{array}\right.
$$

Such a $W_{n}$ is named the $W$-mapping generated by $S_{n}, S_{n-1}, \ldots, S_{0}$ and $\lambda_{n}, \lambda_{n-1}, \ldots, \lambda_{0}$; see [30]. 
Lemma 9 ([30]). Let $C$ be a convex closed nonempty set in a Hilbert space $H$. Let $\left\{S_{n}\right\}_{n=0}^{\infty}$ be a mapping sequence of nonexpansivity on $C$ with $\bigcap_{n=0}^{\infty} \operatorname{Fix}\left(S_{n}\right) \neq \varnothing$. Let $\left\{\lambda_{n}\right\}_{n=0}^{\infty}$ be a number sequence in $(0, b]$ for some $b \in(0,1)$. Then $\lim _{n \rightarrow \infty} U_{n, k} x$ exists for every $x \in C$ and $k \geq 0$.

Using Lemma 9, $W: C \rightarrow C$ is defined by $W x=\lim _{n \rightarrow \infty} W_{n} x=\lim _{n \rightarrow \infty} U_{n, 0} x, \forall x \in C$. We call $W$ is the $W$-mapping defined by $\left\{S_{n}\right\}_{n=0}^{\infty}$ and $\left\{\lambda_{n}\right\}_{n=0}^{\infty}$. Next, we assume that $\left\{\lambda_{n}\right\}_{n=0}^{\infty}$ is a sequence of positive numbers in $(0, b]$ for some $b \in(0,1)$.

Lemma 10 ([30]). Let $C$ be a convex closed nonempty set of a Hilbert space $H$. Let $\left\{S_{n}\right\}_{n=0}^{\infty}$ be a mapping sequence of nonexpansivity on $C$ with $\bigcap_{n=0}^{\infty} \operatorname{Fix}\left(S_{n}\right) \neq \varnothing$. Let $\left\{\lambda_{n}\right\}_{n=0}^{\infty}$ be a number sequence in $(0, b]$ for some $b \in(0,1)$. Then $\bigcap_{n=0}^{\infty} \operatorname{Fix}\left(S_{n}\right)=\operatorname{Fix}(W)$.

Lemma 11 ([30]). Let $C$ be a convex closed nonempty set of a Hilbert space $H$. Let $\left\{S_{n}\right\}_{n=0}^{\infty}$ be a sequence of nonexpansive self-mappings on $C$ with $\bigcap_{n=0}^{\infty} \operatorname{Fix}\left(S_{n}\right) \neq \varnothing$, and $\left\{\lambda_{n}\right\}_{n=0}^{\infty}$ be a real sequence in $(0, b]$ for some $b \in(0,1)$. If $D$ is any bounded subset of $C$, then $\lim _{n \rightarrow \infty} \sup _{x \in D}\left\|W_{n} x-W x\right\|=0$.

Lemma 12 ([21]). Let $C$ be a convex closed nonempty set of a Hilbert space $H$. Let $A: C \rightarrow H$ be a hemicontinuous nonself monotone mapping. Then the following hold: (i) $\operatorname{VI}(C, A)=\left\{x^{*} \in C:\left\langle x^{*}-y, A y\right\rangle \leq\right.$ $0 \forall y \in C\} ;($ ii $) \operatorname{VI}(C, A)=\operatorname{Fix}\left(P_{C}(I-\lambda A)\right)$ for all $\lambda>0$; (iii) $\operatorname{VI}(C, A)$ consists of one point, if $A$ is strongly monotone and Lipschitz continuous.

\section{Main Results}

Let $C$ be a convex closed nonempty set of a real Hilbert space $H$. Let the mappings $A_{1}, B_{i}: C \rightarrow H$ be monotone for $i=1,2$. Let $T: C \rightarrow C$ be an asymptotically nonexpansive self mapping and $\left\{S_{n}\right\}_{n=0}^{\infty}$ be a countable family of nonexpansive self mappings on $C$. We now consider the variational inequality for mapping $A_{1}$ over the common solution set $\Omega$ of the GSVI (1) and the CFPP of $\left\{S_{n}\right\}_{n=0}^{\infty}$ and T:

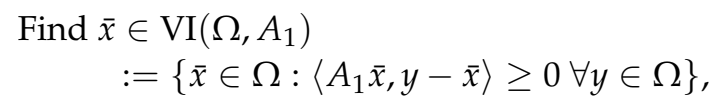

where $\Omega:=\bigcap_{n=0}^{\infty} \operatorname{Fix}\left(S_{n}\right) \cap \operatorname{GSVI}\left(C, B_{1}, B_{2}\right) \cap \operatorname{Fix}(T) \neq \varnothing$. This section introduces the following general monotone variational inequality with the variational inequality constraint on the common solution set of the GSVI (1) and the CFPP of $\left\{S_{n}\right\}_{n=0}^{\infty}$ and T, which is named as the triple hierarchical constrained variational inequality (THCVI):

Problem 2. Assume that

(C1) $T: C \rightarrow C$ is an asymptotically nonexpansive self mapping with a sequence $\left\{\theta_{n}\right\} \subset[0,+\infty)$;

(C2) $\left\{S_{n}\right\}_{n=0}^{\infty}$ is a countable family of nonexpansive self mappings on $C$;

(C3) $B_{1}, B_{2}: C \rightarrow H$ are $\alpha$-inverse-strongly monotone and $\beta$-inverse-strongly monotone, respectively;

(C4) $\operatorname{GSVI}\left(C, B_{1}, B_{2}\right):=\operatorname{Fix}(G)$ where $G:=P_{C}\left(P_{C}\left(I-\mu_{2} B_{2}\right)-\mu_{1} B_{1} P_{C}\left(I-\mu_{2} B_{2}\right)\right)$ for real numbers $\mu_{1}, \mu_{2}>0$

(C5) $\Omega:=\bigcap_{n=0}^{\infty} \operatorname{Fix}\left(S_{n}\right) \cap \operatorname{GSVI}\left(C, B_{1}, B_{2}\right) \cap \operatorname{Fix}(T) \neq \varnothing$;

(C6) $W_{n}$ is the $W$-mapping defined by $S_{n}, S_{n-1}, \ldots, S_{0}$ and $\lambda_{n}, \lambda_{n-1}, \ldots, \lambda_{0}$, where $\left\{\lambda_{n}\right\}_{n=0}^{\infty} \subset(0,1)$;

(C7) $A_{1}: C \rightarrow H$ is $\zeta$-inverse-strongly monotone;

(C8) $A_{2}: C \rightarrow H$ is $\eta$-strongly monotone and $\kappa$-Lipschitzian;

(C9) $f: C \rightarrow C$ is a $\delta$-contraction mapping with real coefficient $\delta \in[0,1)$;

(C10) $\operatorname{VI}\left(\Omega, A_{1}\right) \neq \varnothing$.

Then the objective is to

$$
\text { find } \begin{aligned}
x^{*} & \in \operatorname{VI}\left(\operatorname{VI}\left(\Omega, A_{1}\right), \mu A_{2}-f\right) \\
& :=\left\{x^{*} \in \operatorname{VI}\left(\Omega, A_{1}\right):\left\langle x^{*}-v,\left(\mu A_{2}-f\right) x^{*}\right\rangle \leq 0 \forall v \in \operatorname{VI}\left(\Omega, A_{1}\right)\right\},
\end{aligned}
$$


for some $\mu>0$.

Problem 3. If we put $f=0$ in Problem 2, then the objective is to

$$
\text { find } \begin{aligned}
x^{*} & \in \operatorname{VI}\left(\operatorname{VI}\left(\Omega, A_{1}\right), A_{2}\right) \\
& :=\left\{x^{*} \in \operatorname{VI}\left(\Omega, A_{1}\right):\left\langle A_{2} x^{*}, v-x^{*}\right\rangle \geq 0 \forall v \in \operatorname{VI}\left(\Omega, A_{1}\right)\right\} .
\end{aligned}
$$

Here we propose the following implicit Mann-type iteration algorithms (Algorithms 2 and 3) for solving Problems 2 and 3, respectively.

\section{Algorithm 2.}

Step 0. Take $\left\{\alpha_{n}\right\}_{n=0}^{\infty},\left\{\beta_{n}\right\}_{n=0}^{\infty},\left\{\gamma_{n}\right\}_{n=0}^{\infty},\left\{\delta_{n}\right\}_{n=0}^{\infty} \subset(0, \infty)$, and $\mu>0$, choose $x_{0} \in C$ arbitrarily, and let $n:=0$.

Step 1. Given $x_{n} \in C$, compute $x_{n+1} \in C$ as

$$
\left\{\begin{array}{l}
u_{n}=\left(1-\gamma_{n}\right) W_{n} u_{n}+\gamma_{n} x_{n} \\
y_{n}=P_{C}\left(I-\delta_{n} A_{1}\right) G u_{n} \\
x_{n+1}=\beta_{n} x_{n}+\left(1-\beta_{n}\right) P_{C}\left[\alpha_{n} f\left(x_{n}\right)+\left(I-\alpha_{n} \mu A_{2}\right) T^{n} y_{n}\right]
\end{array}\right.
$$

Update $n:=n+1$ and go to Step 1 .

\section{Algorithm 3.}

Step 0. Take $\left\{\alpha_{n}\right\}_{n=0}^{\infty},\left\{\beta_{n}\right\}_{n=0}^{\infty},\left\{\gamma_{n}\right\}_{n=0}^{\infty},\left\{\delta_{n}\right\}_{n=0}^{\infty} \subset(0, \infty)$, and $\mu>0$, choose $x_{0} \in C$ arbitrarily, and let $n:=0$.

Step 1. Given $x_{n} \in C$, compute $x_{n+1} \in C$ as

$$
\left\{\begin{array}{l}
u_{n}=\left(1-\gamma_{n}\right) W_{n} z_{n}+\gamma_{n} x_{n} \\
v_{n}=P_{C}\left(u_{n}-\mu_{2} B_{2} u_{n}\right) \\
z_{n}=P_{C}\left(v_{n}-\mu_{1} B_{1} v_{n}\right) \\
y_{n}=P_{C}\left(z_{n}-\delta_{n} A_{1} z_{n}\right) \\
x_{n+1}=\beta_{n} x_{n}+\left(1-\beta_{n}\right) P_{C}\left(I-\alpha_{n} \mu A_{2}\right) T^{n} y_{n}
\end{array}\right.
$$

Update $n:=n+1$ and go to Step 1 .

We are now able to state and prove the main results of this paper: the following convergence analysis is presented for our Algorithms 2 and 3.

Theorem 1. Assume that $\mu_{1}$ is a real number in $(0,2 \alpha)$, and $\mu_{2}$ is a real number in $(0,2 \beta)$. Let $\delta<\tau:=$ $1-\sqrt{1-\mu\left(2 \eta-\mu \kappa^{2}\right)} \in(0,1]$ for $\mu \in\left(0, \frac{2 \eta}{\kappa^{2}}\right)$. We suppose $\left\{\lambda_{n}\right\}_{n=0}^{\infty}$ is a real sequence in $(0, b]$ for some real number $b$ in $(0,1)$. We also suppose that $\left\{\alpha_{n}\right\},\left\{\beta_{n}\right\},\left\{\gamma_{n}\right\} \subset(0,1]$ and $\left\{\delta_{n}\right\} \subset(0,2 \zeta]$ such that

(i) $\sum_{n=0}^{\infty} \alpha_{n}=\infty$ and $\lim _{n \rightarrow \infty} \alpha_{n}=0$;

(ii) $\delta_{n} \leq \alpha_{n} \forall n \geq 0$ and $\lim _{n \rightarrow \infty} \frac{\theta_{n}}{\alpha_{n}}=0$;

(iii) $\liminf _{n \rightarrow \infty} \beta_{n}>0$ and $\lim \sup _{n \rightarrow \infty} \beta_{n}<1$;

(iv) $\liminf _{n \rightarrow \infty} \gamma_{n}>0$, $\limsup _{n \rightarrow \infty} \gamma_{n}<1$ and $\lim _{n \rightarrow \infty}\left|\gamma_{n+1}-\gamma_{n}\right|=0$;

(v) $\lim _{n \rightarrow \infty}\left\|T^{n+1} y_{n}-T^{n} y_{n}\right\|=0$.

Then the sequence $\left\{x_{n}\right\}_{n=0}^{\infty}$ generated by Algorithm 2 satisfies the following properties:

(a) $\left\{x_{n}\right\}_{n=0}^{\infty}$ is bounded;

(b) $\lim _{n \rightarrow \infty}\left\|x_{n}-y_{n}\right\|=0, \lim _{n \rightarrow \infty}\left\|x_{n}-G x_{n}\right\|=0, \lim _{n \rightarrow \infty}\left\|x_{n}-T x_{n}\right\|=0$ and $\lim _{n \rightarrow \infty} \| x_{n}-$ $W x_{n} \|=0$;

(c) if $\lim _{n \rightarrow} \frac{\left\|x_{n}-y_{n}\right\|}{\delta_{n}}=0$, then $x_{n} \rightarrow x^{*} \in \operatorname{VI}\left(\Omega, A_{1}\right)$. 
Proof. First of all, for any $x, y \in C$, by Lemma 4, we have

$$
\left\|P_{\mathrm{VI}\left(\Omega, A_{1}\right)}\left(f+I-\mu A_{2}\right) x-P_{\mathrm{VI}\left(\Omega, A_{1}\right)}\left(f+I-\mu A_{2}\right) y\right\| \leq \delta\|x-y\|+(1-\tau)\|x-y\|=[1-(\tau-\delta)]\|x-y\|
$$

which implies that $P_{\mathrm{VI}\left(\Omega, A_{1}\right)}\left(f+I-\mu A_{2}\right)$ is a contraction. Banach's Contraction Principle tells us that $P_{\mathrm{VI}\left(\Omega, A_{1}\right)}\left(f+I-\mu A_{2}\right)$ has a fixed point. Indeed, it is also unique, say $x^{*} \in C$, that is, $x^{*}=$ $P_{\mathrm{VI}\left(\Omega, A_{1}\right)}\left(f+I-\mu A_{2}\right) x^{*}$. Utilizing Lemma 12, we get

$$
\left\{x^{*}\right\}=\operatorname{Fix}\left(P_{\mathrm{VI}\left(\Omega, A_{1}\right)}\left(f+I-\mu A_{2}\right)\right)=\mathrm{VI}\left(\mathrm{VI}\left(\Omega, A_{1}\right), \mu A_{2}-f\right) .
$$

That is, the Problem 2 has the unique solution. Since $\lim \inf _{n \rightarrow \infty} \gamma_{n}>0$ and $\limsup _{n \rightarrow \infty} \gamma_{n}<1$, we can suppose that $\left\{\gamma_{n}\right\} \subset\left[a_{0}, b_{0}\right]$ is subset of $(0,1)$ for some $a_{0}, b_{0} \in(0,1)$. Since $G$ : is defined from $C$ to $C$ as $G:=P_{C}\left(P_{C}\left(I-\mu_{2} B_{2}\right)-\mu_{1} B_{1} P_{C}\left(I-\mu_{2} B_{2}\right)\right)$. Here $\mu_{1} \in(0,2 \alpha)$ and $\mu_{2} \in(0,2 \beta), G$ is nonexpansive by Lemma 6 . It is easy to see that for each $n \geq 0$ there exists a unique element $u_{n} \in C$ such that

$$
u_{n}=\gamma_{n} x_{n}+\left(1-\gamma_{n}\right) W_{n} u_{n}
$$

As a matter of fact, we utilize $F_{n} x:=\gamma_{n} x_{n}+\left(1-\gamma_{n}\right) W_{n} x \forall x \in C$. Since each $W_{n}: C \rightarrow C$ is a nonexpansive mapping, we get

$$
\left\|F_{n} x-F_{n} y\right\|=\left(1-\gamma_{n}\right)\left\|W_{n} x-W_{n} y\right\| \leq\left(1-\gamma_{n}\right)\|x-y\|, \quad \forall x, y \in C
$$

Also, from $\left\{\gamma_{n}\right\} \subset\left[a_{0}, b_{0}\right]$ and $\left[a_{0}, b_{0}\right] \subset(0,1)$ we have $0<1-\gamma_{n}<1, \forall n \geq 0$. Thus, $F_{n}: C \rightarrow C$ is a contraction. Banach's Contraction Principle infers there exists a unique element $u_{n}$ in set $C$ satisfying (3).

Here, we are able to divide the rest of the proof into several steps.

Step 1. We claim that all the vector sequences $\left\{x_{n}\right\},\left\{y_{n}\right\},\left\{z_{n}\right\},\left\{u_{n}\right\},\left\{v_{n}\right\},\left\{T^{n} y_{n}\right\}$ and $\left\{A_{2}\left(T^{n} y_{n}\right)\right\}$ are bounded, where $v_{n}=P_{C}\left(u_{n}-\mu_{2} B_{2} u_{n}\right)$ and $z_{n}=P_{C}\left(v_{n}-\mu_{1} B_{1} v_{n}\right)$ for all $n \geq 0$. Indeed, it is clear that (2) can be rewritten as

$$
\left\{\begin{array}{l}
u_{n}=\left(1-\gamma_{n}\right) W_{n} u_{n}+\gamma_{n} x_{n} \\
z_{n}=G u_{n} \\
y_{n}=P_{C}\left(I-\delta_{n} A_{1}\right) z_{n} \\
x_{n+1}=\left(1-\beta_{n}\right) P_{C}\left[\left(I-\alpha_{n} \mu A_{2}\right) T^{n} y_{n}+\alpha_{n} f\left(x_{n}\right)\right]+\beta_{n} x_{n}
\end{array}\right.
$$

Take an arbitrary

$$
p \in \Omega=\bigcap_{n=0}^{\infty} \operatorname{Fix}\left(S_{n}\right) \cap \operatorname{GSVI}\left(C, B_{1}, B_{2}\right) \cap \operatorname{Fix}(T) .
$$

Then $p=W_{n} p, p=T p$ and $p=G p$. Since each $W_{n}: C \rightarrow C$ is nonexpansive, (4) infers to

$$
\left\|u_{n}-p\right\| \leq\left(1-\gamma_{n}\right)\left\|u_{n}-p\right\|+\gamma_{n}\left\|x_{n}-p\right\|
$$

which hence yields

$$
\left\|u_{n}-p\right\| \leq\left\|x_{n}-p\right\|, \quad \forall n \geq 0 .
$$

It is easy to infer from (4) that

$$
\left\|z_{n}-p\right\|=\left\|G u_{n}-p\right\| \leq\left\|u_{n}-p\right\| \leq\left\|x_{n}-p\right\|, \quad \forall n \geq 0
$$


Since $\liminf _{n \rightarrow \infty} \beta_{n}>0$ and $\limsup _{n \rightarrow \infty} \beta_{n}<1$, we suppose that $\left\{\beta_{n}\right\} \subset[c, d]$. Since $\lim _{n \rightarrow \infty} \frac{\theta_{n}}{\alpha_{n}}=0$, we can also suppose that

$$
\theta_{n} \leq \frac{\alpha_{n}(\tau-\delta)(1-d)}{2} \leq \alpha_{n}(\tau-\delta) \text {. }
$$

Note that $\delta_{n} \leq \alpha_{n}, \forall n \geq 0 . \zeta$-inverse-strong monotonicity of $A_{1}$ and Lemma 5 yield

$$
\left\|y_{n}-p\right\| \leq\left\|\left(I-\delta_{n} A_{1}\right) z_{n}-\left(I-\delta_{n} A_{1}\right) p-\delta_{n} A_{1} p\right\| \leq \delta_{n}\left\|A_{1} p\right\|+\left\|z_{n}-p\right\| \leq\left\|x_{n}-p\right\|+\delta_{n}\left\|A_{1} p\right\| .
$$

Utilizing Lemma 4 and (7), we obtain from (4) that

$$
\begin{aligned}
& \left\|x_{n+1}-p\right\| \\
\leq & \left(1-\beta_{n}\right)\left\|\alpha_{n}\left(f\left(x_{n}\right)-\mu A_{2} p\right)+\left(I-\alpha_{n} \mu A_{2}\right) T^{n} y_{n}-\left(I-\alpha_{n} \mu A_{2}\right) p\right\|+\beta_{n}\left\|x_{n}-p\right\| \\
\leq & \left(1-\beta_{n}\right)\left[\alpha_{n} \delta\left\|x_{n}-p\right\|+\alpha_{n}\left\|f(p)-\mu A_{2} p\right\|+\left(1-\alpha_{n} \tau\right)\left(1+\theta_{n}\right)\left\|y_{n}-p\right\|\right]+\beta_{n}\left\|x_{n}-p\right\| \\
\leq & \beta_{n}\left\|x_{n}-p\right\|+\left(1-\beta_{n}\right)\left\{\alpha_{n} \delta\left\|x_{n}-p\right\|+\alpha_{n}\left\|\mu A_{2} p-f(p)\right\|\right. \\
& \left.+\left(1-\alpha_{n} \tau\right)\left[\left\|x_{n}-p\right\|+\delta_{n}\left\|A_{1} p\right\|\right]+\theta_{n}\left[\delta_{n}\left\|A_{1} p\right\|+\left\|x_{n}-p\right\|\right]\right\} \\
\leq & \beta_{n}\left\|x_{n}-p\right\|+\left(1-\beta_{n}\right)\left\{\alpha_{n} \delta\left\|x_{n}-p\right\|+\alpha_{n}\left\|\mu A_{2} p-f(p)\right\|\right. \\
& \left.+\left(1-\alpha_{n} \tau\right)\left[\left\|x_{n}-p\right\|+\delta_{n}\left\|A_{1} p\right\|\right]+\theta_{n}\left\|x_{n}-p\right\|+(\tau-\delta) \alpha_{n} \delta_{n}\left\|A_{1} p\right\|\right\} \\
\leq & {\left[1-\alpha_{n}\left(1-\beta_{n}\right)(\tau-\delta)\right]\left\|x_{n}-p\right\|+\theta_{n}\left\|x_{n}-p\right\|+\alpha_{n}\left\|\mu A_{2} p-f(p)\right\|+\alpha_{n}\left\|A_{1} p\right\| } \\
\leq & {\left[1-\frac{\alpha_{n}(1-d)(\tau-\delta)}{2}\right]\left\|x_{n}-p\right\|+\frac{\alpha_{n}(1-d)(\tau-\delta)}{2} \cdot \frac{2\left(\left\|A_{1} p\right\|+\left\|\mu A_{2} p-f(p)\right\|\right)}{(1-d)(\tau-\delta)} } \\
\leq & \max \left\{\frac{2\left(\left\|A_{1} p\right\|+\left\|\mu A_{2} p-f(p)\right\|\right)}{(1-d)(\tau-\delta)},\left\|x_{n}-p\right\|\right\} .
\end{aligned}
$$

By simple induction, we have

$$
\left\|x_{n+1}-p\right\| \leq \max \left\{\frac{2\left(\left\|f(p)-\mu A_{2} p\right\|\right)+\left\|A_{1} p\right\|}{(1-d)(\tau-\delta)},\left\|x_{0}-p\right\|\right\}, \quad \forall n \geq 0 .
$$

Therefore, $\left\{x_{n}\right\}$ is a bounded vector sequence, and so are all the other sequences $\left\{y_{n}\right\},\left\{z_{n}\right\},\left\{u_{n}\right\},\left\{T^{n} y_{n}\right\}$ and $\left\{A_{2}\left(T^{n} y_{n}\right)\right\}$ (due to the Lipschitz continuity of $T$ and $A_{2}$ ). Since each $W_{n}$ enjoys the nonexpansivity on $C$, we get

$$
\left\|W_{n} u_{n}\right\| \leq\left\|W_{n} u_{n}-p\right\|+\|p\| \leq\left\|u_{n}-p\right\|+\|p\|,
$$

which yields that $\left\{W_{n} u_{n}\right\}$ is bounded too. In addition, from Lemma 2 and $p$ is a element in $\Omega \subset$ $\operatorname{GSVI}\left(C, B_{1}, B_{2}\right)$, it also follows that $(p, q)$ is a solution of GSVI (1) where $q=P_{C}\left(I-\mu_{2} B_{2}\right) p$. Note that $v_{n}=P_{C}\left(I-\mu_{2} B_{2}\right) u_{n}$ for all $n \geq 0$. Then by Lemma 5 , we get

$$
\left\|v_{n}\right\| \leq\left\|P_{C}\left(I-\mu_{2} B_{2}\right) u_{n}-P_{C}\left(I-\mu_{2} B_{2}\right) p\right\|+\|q\| \leq\left\|u_{n}-p\right\|+\|q\| .
$$

This yields vector sequence $\left\{v_{n}\right\}$ is bounded.

Step 2. We claim that $\left\|x_{n}-x_{n+1}\right\| \rightarrow 0$ and $\left\|y_{n}-y_{n+1}\right\| \rightarrow 0$ as $n \rightarrow \infty$. Indeed, we set $x_{n+1}=\beta_{n} x_{n}+\left(1-\beta_{n}\right) w_{n}, \forall n \geq 0$. Then $w_{n}=P_{C}\left[\left(I-\alpha_{n} \mu A_{2}\right) T^{n} y_{n}+\alpha_{n} f\left(x_{n}\right)\right]$. It follows from (4) that

$$
\begin{aligned}
\left\|w_{n+1}-w_{n}\right\| \leq & \left\|\alpha_{n+1} f\left(x_{n+1}\right)+\left(I-\alpha_{n+1} \mu A_{2}\right) T^{n+1} y_{n+1}-\alpha_{n} f\left(x_{n}\right)-\left(I-\alpha_{n} \mu A_{2}\right) T^{n} y_{n}\right\| \\
\leq & \left\|T^{n+1} y_{n+1}-T^{n+1} y_{n}\right\|+\left\|T^{n+1} y_{n}-T^{n} y_{n}\right\|+\alpha_{n+1}\left\|\mu A_{2}\left(T^{n+1} y_{n+1}\right)\right\| \\
& +\alpha_{n}\left\|\mu A_{2}\left(T^{n} y_{n}\right)\right\|+\alpha_{n+1}\left\|f\left(x_{n+1}\right)\right\|+\alpha_{n}\left\|f\left(x_{n}\right)\right\| \\
\leq & \left(1+\theta_{n+1}\right)\left\|y_{n+1}-y_{n}\right\|+\left\|T^{n+1} y_{n}-T^{n} y_{n}\right\|+\alpha_{n+1}\left(\left\|f\left(x_{n+1}\right)\right\|\right. \\
& \left.+\left\|\mu A_{2}\left(T^{n+1} y_{n+1}\right)\right\|\right)+\alpha_{n}\left(\left\|f\left(x_{n}\right)\right\|+\left\|\mu A_{2}\left(T^{n} y_{n}\right)\right\|\right) .
\end{aligned}
$$


Since vector sequence $\left\{\delta_{n}\right\}$ falls into $(0,2 \zeta]$ and $A_{1}$ is $\zeta$-inverse-strongly monotone, by Lemma 5 we obtain

$$
\begin{aligned}
\left\|y_{n+1}-y_{n}\right\| & \leq\left\|\left(z_{n+1}-\delta_{n+1} A_{1} z_{n+1}\right)-\left(z_{n}-\delta_{n} A_{1} z_{n}\right)\right\| \\
& \leq\left\|\left(z_{n+1}-\delta_{n+1} A_{1} z_{n+1}\right)-\left(z_{n}-\delta_{n+1} A_{1} z_{n}\right)\right\|+\left|\delta_{n}-\delta_{n+1}\right|\left\|A_{1} z_{n}\right\| \\
& \leq\left\|u_{n+1}-u_{n}\right\|+\left|\delta_{n}-\delta_{n+1}\right|\left\|A_{1} z_{n}\right\| .
\end{aligned}
$$

Since simple calculations show that

$$
\begin{aligned}
\left\|u_{n} u_{n+1}\right\| \leq & \gamma_{n+1}\left\|x_{n}-x_{n+1}\right\|+\left(1-\gamma_{n+1}\right)\left\|W_{n} u_{n}-W_{n+1} u_{n+1}\right\| \\
& +\left|\gamma_{n}-\gamma_{n+1}\right|\left\|W_{n} u_{n}-x_{n}\right\| \\
\leq & \gamma_{n+1}\left\|x_{n}-x_{n+1}\right\|+\left(1-\gamma_{n+1}\right)\left[\left\|W_{n} u_{n+1}-W_{n+1} u_{n+1}\right\|\right. \\
& \left.+\left\|W_{n} u_{n}-W_{n} u_{n+1}\right\|\right]+\left|\gamma_{n}-\gamma_{n+1}\right|\left\|W_{n} u_{n}-x_{n}\right\| \\
\leq & \left(1-\gamma_{n+1}\right)\left[\left\|W_{n} u_{n+1}-W_{n+1} u_{n+1}\right\|+\gamma_{n+1}\left\|x_{n}-x_{n+1}\right\|\right. \\
& \left.+\left\|u_{n}-u_{n+1}\right\|\right]+\left|\gamma_{n}-\gamma_{n+1}\right|\left\|W_{n} u_{n}-x_{n}\right\|,
\end{aligned}
$$

it follows that

$$
a_{0}\left\|u_{n}-u_{n+1}\right\| \leq a_{0}\left\|x_{n+1}-x_{n}\right\|+\left\|W_{n+1} u_{n+1}-W_{n} u_{n+1}\right\|+a_{0}\left\|x_{n}-W_{n} u_{n}\right\|\left|\gamma_{n+1}-\gamma_{n}\right|
$$

Since $D:=\left\{u_{n}: n \geq 0\right\} \subset C$ is bounded subset, by the argument process in Lemma 11 we get $\sum_{n=0}^{\infty} \sup _{x \in D}\left\|W_{n+1} x-W_{n} x\right\|<\infty$. Thus we have

$$
\sum_{n=0}^{\infty}\left\|W_{n+1} u_{n+1}-W_{n} u_{n+1}\right\|<\infty .
$$

Therefore, from (8)-(10) we deduce that

$$
\begin{aligned}
& \left\|w_{n}-w_{n+1}\right\| \\
& \leq\left|\delta_{n}-\delta_{n+1}\right|\left\|A_{1} z_{n}\right\|+\theta_{n+1}\left\|y_{n}-y_{n+1}\right\|+\left\|T^{n} y_{n}-T^{n+1} y_{n}\right\|+\left\|u_{n}-u_{n+1}\right\| \\
& \quad+\alpha_{n+1}\left(\left\|f\left(x_{n+1}\right)\right\|+\left\|\mu A_{2}\left(T^{n+1} y_{n+1}\right)\right\|\right)+\alpha_{n}\left(\left\|f\left(x_{n}\right)\right\|+\left\|\mu A_{2}\left(T^{n} y_{n}\right)\right\|\right) \\
& \leq \frac{1}{a_{0}}\left\|W_{n} u_{n+1}-W_{n+1} u_{n+1}\right\|+\left\|x_{n+1}-x_{n}\right\|+\left|\gamma_{n+1}-\gamma_{n}\right| \frac{\left\|W_{n} u_{n}-x_{n}\right\|}{a_{0}}+\left|\delta_{n}-\delta_{n+1}\right|\left\|A_{1} z_{n}\right\| \\
& \quad+\theta_{n+1}\left\|y_{n}-y_{n+1}\right\|+\left\|T^{n} y_{n}-T^{n+1} y_{n}\right\|+\alpha_{n+1}\left(\left\|f\left(x_{n+1}\right)\right\|+\left\|\mu A_{2}\left(T^{n+1} y_{n+1}\right)\right\|\right. \\
& \quad+\alpha_{n} \|\left(\left\|f\left(x_{n}\right)\right\|+\mu A_{2}\left(T^{n} y_{n}\right) \|\right),
\end{aligned}
$$

which immediately attains

$$
\begin{aligned}
& \left\|w_{n}-w_{n+1}\right\|-\left\|x_{n}-x_{n+1}\right\| \\
& \leq \frac{1}{a_{0}}\left\|W_{n} u_{n+1}-W_{n+1} u_{n+1}\right\|+\left|\gamma_{n}-\gamma_{n+1}\right| \frac{\left\|W_{n} u_{n}-x_{n}\right\|}{a_{0}}+\left|\delta_{n}-\delta_{n+1}\right|\left\|A_{1} z_{n}\right\| \\
& \quad+\theta_{n+1}\left\|y_{n}-y_{n+1}\right\|+\left\|T^{n} y_{n}-T^{n+1} y_{n}\right\|+\alpha_{n+1}\left(\left\|f\left(x_{n+1}\right)\right\|+\left\|\mu A_{2}\left(T^{n+1} y_{n+1}\right)\right\|\right) \\
& \quad+\alpha_{n}\left(\left\|f\left(x_{n}\right)\right\|+\left\|\mu A_{2}\left(T^{n} y_{n}\right)\right\|\right) .
\end{aligned}
$$

Since

$$
\lim _{n \rightarrow \infty}\left\|T^{n} y_{n}-T^{n+1} y_{n}\right\|=\lim _{n \rightarrow \infty} \theta_{n}=0,
$$

from (11) and conditions (i), (ii), (iv) we get $\limsup _{n \rightarrow \infty}\left(\left\|w_{n}-w_{n+1}\right\|-\left\|x_{n}-x_{n+1}\right\|\right) \leq 0$. Hence, by condition (iii) and Lemma 8 , we get $\lim _{n \rightarrow \infty}\left\|w_{n}-x_{n}\right\|=0$. Consequently,

$$
\lim _{n \rightarrow \infty}\left(1-\beta_{n}\right)\left\|w_{n}-x_{n}\right\|=\lim _{n \rightarrow \infty}\left\|x_{n}-x_{n+1}\right\|=0
$$


Again from (9) and (10) we conclude that

$$
\begin{aligned}
& a_{0}\left\|y_{n}-y_{n+1}\right\| \\
& \leq a_{0}\left\|x_{n}-x_{n+1}\right\|+\left\|W_{n} u_{n+1}-W_{n+1} u_{n+1}\right\|+a_{0}\left|\gamma_{n}-\gamma_{n+1}\right| \\
& \left\|W_{n} u_{n}-x_{n}\right\|+\left|\delta_{n}-\delta_{n+1}\right| a_{0}\left\|A_{1} z_{n}\right\| \rightarrow 0
\end{aligned}
$$

and $\left\|z_{n+1}-z_{n}\right\|=\left\|G u_{n+1}-G u_{n}\right\| \leq\left\|u_{n+1}-u_{n}\right\| \rightarrow 0$. Thus,

$$
\lim _{n \rightarrow \infty}\left\|y_{n}-y_{n+1}\right\|=\lim _{n \rightarrow \infty}\left\|u_{n}-u_{n+1}\right\|=\lim _{n \rightarrow \infty}\left\|z_{n}-z_{n+1}\right\|=0
$$

Step 3. We claim that $\lim _{n \rightarrow \infty}\left\|G x_{n}-x_{n}\right\|=0$ as $n \rightarrow \infty$. Indeed, noticing $w_{n}=P_{C}[(I-$ $\left.\left.\alpha_{n} \mu A_{2}\right) T^{n} y_{n}+\alpha_{n} f\left(x_{n}\right)\right] \forall n \geq 0$, we obtain from Lemma 2 that for each $p \in \Omega$,

$$
\left\langle p-w_{n},\left(I-\alpha_{n} \mu A_{2}\right) T^{n} y_{n}+\alpha_{n} f\left(x_{n}\right)-P_{C}\left[\alpha_{n} f\left(x_{n}\right)+\left(I-\alpha_{n} \mu A_{2}\right) T^{n} y_{n}\right]\right\rangle \leq 0 .
$$

From (15), we have

$$
\begin{aligned}
\left\|w_{n}-p\right\|^{2}= & \left\langle P_{C}\left[\left(I-\alpha_{n} \mu A_{2}\right) T^{n} y_{n}+\alpha_{n} f\left(x_{n}\right)\right]-\alpha_{n} f\left(x_{n}\right)-\left(I-\alpha_{n} \mu A_{2}\right) T^{n} y_{n}, w_{n}-p\right\rangle \\
& +\left\langle\left(I-\alpha_{n} \mu A_{2}\right) T^{n} y_{n}+\alpha_{n} f\left(x_{n}\right)-p, w_{n}-p\right\rangle \\
\leq & \left\langle\left(I-\alpha_{n} \mu A_{2}\right) T^{n} y_{n}+\alpha_{n} f\left(x_{n}\right)-p, w_{n}-p\right\rangle \\
= & \left\langle w_{n}-p,\left(I-\alpha_{n} \mu A_{2}\right) T^{n} y_{n}-\left(I-\alpha_{n} \mu A_{2}\right) p\right\rangle+\alpha_{n}\left\langle f\left(x_{n}\right)-\mu A_{2} p, w_{n}-p\right\rangle \\
\leq & {\left[\left(1-\alpha_{n} \tau\right)\left\|T^{n} y_{n}-p\right\|+\delta \alpha_{n}\left\|x_{n}-p\right\|\right]\left\|w_{n}-p\right\|+\alpha_{n}\left\langle w_{n}-p, f(p)-\mu A_{2} p\right\rangle } \\
\leq & \frac{\left[\left(1-\alpha_{n} \tau\right)\left\|T^{n} y_{n}-p\right\|+\alpha_{n} \delta\left\|x_{n}-p\right\|\right]^{2}}{2}+\frac{1}{2}\left\|w_{n}-p\right\|^{2}+\alpha_{n}\left\langle w_{n}-p, f(p)-\mu A_{2} p\right\rangle,
\end{aligned}
$$

which leads to

$$
\begin{aligned}
& \left\|w_{n}-p\right\|^{2} \\
& \leq\left(1-\alpha_{n} \tau\right)\left\|T^{n} y_{n}-p\right\|^{2}+\delta \alpha_{n}\left\|x_{n}-p\right\|^{2}-2 \alpha_{n}\left\langle w_{n}-p, \mu A_{2} p-f(p)\right\rangle \\
& \leq\left(1-\alpha_{n} \tau\right)\left(1+\theta_{n}\right)^{2}\left\|y_{n}-p\right\|^{2}+\alpha_{n} \delta\left\|x_{n}-p\right\|^{2}-2 \alpha_{n}\left\langle w_{n}-p, \mu A_{2} p-f(p)\right\rangle \\
& \leq\left(1-\alpha_{n} \tau\right)\left\|y_{n}-p\right\|^{2}+\alpha_{n} \delta\left\|x_{n}-p\right\|^{2}+\theta_{n}\left(2+\theta_{n}\right)\left\|y_{n}-p\right\|^{2}-2 \alpha_{n}\left\langle w_{n}-p, \mu A_{2} p-f(p)\right\rangle .
\end{aligned}
$$

From (7) and (16), we get

$$
\begin{aligned}
& \left\|x_{n+1}-p\right\|^{2} \\
\leq & \beta_{n}\left\|x_{n}-p\right\|^{2}+\left(1-\beta_{n}\right)\left[\alpha_{n} \delta\left\|x_{n}-p\right\|^{2}+\left(1-\alpha_{n} \tau\right)\left\|y_{n}-p\right\|^{2}+\theta_{n}\left(2+\theta_{n}\right)\left\|y_{n}-p\right\|^{2}\right. \\
& \left.+2 \alpha_{n}\left\langle f(p)-\mu A_{2} p, w_{n}-p\right\rangle\right] \\
\leq & \beta_{n}\left\|x_{n}-p\right\|^{2}+\left(1-\beta_{n}\right)\left\{\alpha_{n} \delta\left\|x_{n}-p\right\|^{2}+\left(1-\alpha_{n} \tau\right)\left(\left\|z_{n}-p\right\|+\delta_{n}\left\|A_{1} p\right\|\right)^{2}\right. \\
& \left.+\theta_{n}\left(2+\theta_{n}\right)\left\|y_{n}-p\right\|^{2}+2 \alpha_{n}\left\langle f(p)-\mu A_{2} p, w_{n}-p\right\rangle\right\} \\
\leq & \beta_{n}\left\|x_{n}-p\right\|^{2}+\left(1-\beta_{n}\right)\left[\alpha_{n} \delta\left\|x_{n}-p\right\|^{2}+\left(1-\alpha_{n} \tau\right)\left\|z_{n}-p\right\|^{2}\right] \\
& +\delta_{n}\left\|A_{1} p\right\|\left(2\left\|z_{n}-p\right\|+\delta_{n}\left\|A_{1} p\right\|\right)+\theta_{n}\left(2+\theta_{n}\right)\left\|y_{n}-p\right\|^{2}+2 \alpha_{n}\left\|f(p)-\mu A_{2} p\right\|\left\|p-w_{n}\right\| .
\end{aligned}
$$

We now note that $q=P_{C}\left(p-\mu_{2} B_{2} p\right), v_{n}=P_{C}\left(u_{n}-\mu_{2} B_{2} u_{n}\right)$ and $z_{n}=P_{C}\left(v_{n}-\mu_{1} B_{1} v_{n}\right)$. Then $z_{n}=G u_{n}$. By Lemma 5 we have

$$
\left\|v_{n}-q\right\|^{2} \leq\left\|u_{n}-p-\mu_{2}\left(B_{2} u_{n}-B_{2} p\right)\right\|^{2} \leq\left\|u_{n}-p\right\|^{2}-\mu_{2}\left(2 \beta-\mu_{2}\right)\left\|B_{2} u_{n}-B_{2} p\right\|^{2}
$$

and

$$
\left\|z_{n}-p\right\|^{2} \leq\left\|v_{n}-q-\mu_{1}\left(B_{1} v_{n}-B_{1} q\right)\right\|^{2} \leq\left\|v_{n}-q\right\|^{2}-\mu_{1}\left(2 \alpha-\mu_{1}\right)\left\|B_{1} v_{n}-B_{1} q\right\|^{2} .
$$


Substituting (18) for (19), we obtain from (5) that

$$
\left\|z_{n}-p\right\|^{2} \leq \mu_{2}\left(\mu_{2}-2 \beta\right)\left\|B_{2} u_{n}-B_{2} p\right\|^{2}+\mu_{1}\left(\mu_{1}+2 \alpha\right)\left\|B_{1} v_{n}-B_{1} q\right\|^{2}+\left\|x_{n}-p\right\|^{2} .
$$

Combining (17) and (20), we get

$$
\begin{aligned}
& \left\|x_{n+1}-p\right\|^{2} \\
& \leq \beta_{n}\left\|x_{n}-p\right\|^{2}+\left(1-\beta_{n}\right)\left\{\alpha_{n} \delta\left\|x_{n}-p\right\|^{2}+\left(1-\tau \alpha_{n}\right)\left[\left\|x_{n}-p\right\|^{2}\right.\right. \\
& \left.\left.\quad-\left(2 \beta-\mu_{2}\right) \mu_{2}\left\|B_{2} p-B_{2} u_{n}\right\|^{2}-\left(2 \alpha-\mu_{1}\right) \mu_{1}\left\|B_{1} q-B_{1} v_{n}\right\|^{2}\right]\right\} \\
& \quad+\delta_{n}\left\|A_{1} p\right\|\left(2\left\|z_{n}-p\right\|+\delta_{n}\left\|A_{1} p\right\|\right)+\left(2+\theta_{n}\right) \theta_{n}\left\|y_{n}-p\right\|^{2}+2 \alpha_{n}\left\|\mu A_{2} p-f(p)\right\|\left\|p-w_{n}\right\| \\
& =\left[1-(\tau-\delta) \alpha_{n}\left(1-\beta_{n}\right)\right]\left\|x_{n}-p\right\|^{2}-\left(1-\alpha_{n} \tau\right)\left(1-\beta_{n}\right)\left[\mu_{2}\left(2 \beta-\mu_{2}\right)\left\|B_{2} p-B_{2} u_{n}\right\|^{2}\right. \\
& \left.\quad+\left(2 \alpha-\mu_{1}\right) \mu_{1}\left\|B_{1} q-B_{1} v_{n}\right\|^{2}\right]+\delta_{n}\left\|A_{1} p\right\|\left(2\left\|z_{n}-p\right\|+\delta_{n}\left\|A_{1} p\right\|\right) \\
& \quad+\left(2+\theta_{n}\right) \theta_{n}\left\|y_{n}-p\right\|^{2}+2 \alpha_{n}\left\|\mu A_{2} p-f(p)\right\|\left\|p-w_{n}\right\|,
\end{aligned}
$$

which immediately yields

$$
\begin{aligned}
& \left(1-\alpha_{n} \tau\right)\left(1-\beta_{n}\right)\left[\mu_{2}\left(2 \beta-\mu_{2}\right)\left\|B_{2} p-B_{2} u_{n}\right\|^{2}+\left(2 \alpha-\mu_{1}\right) \mu_{1}\left\|B_{1} q-B_{1} v_{n}\right\|^{2}\right] \\
& \leq\left\|x_{n}-p\right\|^{2}-\left\|x_{n+1}-p\right\|^{2}+\delta_{n}\left\|A_{1} p\right\|\left(\delta_{n}\left\|A_{1} p\right\|+2\left\|z_{n}-p\right\|\right) \\
& \quad+\left(2+\theta_{n}\right) \theta_{n}\left\|y_{n}-p\right\|^{2}+2 \alpha_{n}\left\|\mu A_{2} p-f(p)\right\|\left\|p-w_{n}\right\| \\
& \leq\left\|x_{n}-x_{n+1}\right\|\left(\left\|x_{n}-p\right\|+\left\|x_{n+1}-p\right\|\right)+\delta_{n}\left\|A_{1} p\right\|\left(\delta_{n}\left\|A_{1} p\right\|+2\left\|z_{n}-p\right\|\right) \\
& \quad+\left(2+\theta_{n}\right) \theta_{n}\left\|y_{n}-p\right\|^{2}+2 \alpha_{n}\left\|p-w_{n}\right\|\left\|f(p)-\mu A_{2} p\right\| .
\end{aligned}
$$

Due to condition (iii), $\liminf _{n \rightarrow \infty}\left(1-\beta_{n}\right)>0, \mu_{1} \in(0,2 \alpha), \mu_{2} \in(0,2 \beta), \lim _{n \rightarrow \infty} \theta_{n}=0$, $\lim _{n \rightarrow \infty} \alpha_{n}=0$ and $\lim _{n \rightarrow \infty} \delta_{n}=0$, we obtain from (13) that

$$
\lim _{n \rightarrow \infty}\left\|B_{2} u_{n}-B_{2} p\right\|=0 \quad \text { and } \quad \lim _{n \rightarrow \infty}\left\|B_{1} v_{n}-B_{1} q\right\|=0 .
$$

On the other hand, from Lemma 2 we have

$$
\begin{aligned}
\left\|v_{n}-q\right\|^{2} & \leq\left\langle v_{n}-q, u_{n}-\left(p-\mu_{2} B_{2} p\right)-\mu_{2} B_{2} u_{n}\right\rangle \\
& \leq \frac{1}{2}\left[\left\|u_{n}-p\right\|^{2}+\left\|v_{n}-q\right\|^{2}-\left\|u_{n}-v_{n}-(p-q)\right\|^{2}\right]+\mu_{2}\left\|v_{n}-q\right\|\left\|B_{2} u_{n}-B_{2} p\right\|,
\end{aligned}
$$

which implies that

$$
\left\|v_{n}-q\right\|^{2} \leq\left\|u_{n}-p\right\|^{2}-\left\|(p-q)-u_{n}+v_{n}\right\|^{2}+2 \mu_{2}\left\|v_{n}-q\right\|\left\|B_{2} u_{n}-B_{2} p\right\| .
$$

In the same way, we derive

$$
\left\|z_{n}-p\right\|^{2} \leq\left\|v_{n}-q\right\|^{2}-\left\|(p-q)-v_{n}+z_{n}\right\|^{2}+2 \mu_{1}\left\|z_{n}-p\right\|\left\|B_{1} v_{n}-B_{1} q\right\| .
$$

Substituting (22) for (23), we deduce from (5) that

$$
\begin{aligned}
\left\|z_{n}-p\right\|^{2} \leq & \left\|x_{n}-p\right\|^{2}-\left\|u_{n}-v_{n}-(p-q)\right\|^{2}-\left\|v_{n}-z_{n}+(p-q)\right\|^{2} \\
& +2 \mu_{2}\left\|B_{2} p-B_{2} u_{n}\right\|\left\|v_{n}-q\right\|+2 \mu_{1}\left\|B_{1} q-B_{1} v_{n}\right\|\left\|z_{n}-p\right\| .
\end{aligned}
$$


Combining (17) and (24), we have

$$
\begin{aligned}
& \left\|x_{n+1}-p\right\|^{2} \\
& \leq \beta_{n}\left\|x_{n}-p\right\|^{2}+\left(1-\beta_{n}\right)\left\{\alpha_{n} \delta\left\|x_{n}-p\right\|^{2}+\left(1-\alpha_{n} \tau\right)\left[\left\|x_{n}-p\right\|^{2}-\left\|p-q-u_{n}+v_{n}\right\|^{2}\right.\right. \\
& \left.\left.\quad-\left\|p-q+v_{n}-z_{n}\right\|^{2}+2 \mu_{1}\left\|z_{n}-p\right\|\left\|B_{1} v_{n}-B_{1} q\right\|+2 \mu_{2}\left\|v_{n}-q\right\|\left\|B_{2} u_{n}-B_{2} p\right\|\right]\right\} \\
& \quad+\delta_{n}\left\|A_{1} p\right\|\left(2\left\|z_{n}-p\right\|+\delta_{n}\left\|A_{1} p\right\|\right)+\left(2+\theta_{n}\right) \theta_{n}\left\|y_{n}-p\right\|^{2}+2 \alpha_{n}\left\|\mu A_{2} p-f(p)\right\|\left\|w_{n}-p\right\| \\
& \leq \\
& \quad\left[1-(\tau-\delta) \alpha_{n}\left(1-\beta_{n}\right)\right]\left\|x_{n}-p\right\|^{2}-\left(1-\alpha_{n} \tau\right)\left(1-\beta_{n}\right)\left[\left\|p-q-u_{n}+v_{n}\right\|^{2}\right. \\
& \left.\quad+\left\|p-q+v_{n}-z_{n}\right\|^{2}\right]+2 \mu_{1}\left\|B_{1} v_{n}-B_{1} q\right\|\left\|z_{n}-p\right\|+2 \mu_{2}\left\|v_{n}-q\right\|\left\|B_{2} p-B_{2} u_{n}\right\| \\
& \quad+\delta_{n}\left\|A_{1} p\right\|\left(\delta_{n}\left\|A_{1} p\right\|+2\left\|z_{n}-p\right\|\right)+\left(2+\theta_{n}\right) \theta_{n}\left\|y_{n}-p\right\|^{2}+2 \alpha_{n}\left\|w_{n}-p\right\|\left\|f(p)-\mu A_{2} p\right\|,
\end{aligned}
$$

which hence yields

$$
\begin{aligned}
& \left(1-\alpha_{n} \tau\right)\left(1-\beta_{n}\right)\left[\left\|p-q-u_{n}+v_{n}\right\|^{2}+\left\|p-q+v_{n}-z_{n}\right\|^{2}\right] \\
& \leq \\
& \quad\left\|x_{n}-p\right\|^{2}-\left\|x_{n+1}-p\right\|^{2}+2 \mu_{2}\left\|v_{n}-q\right\|\left\|B_{2} p-B_{2} u_{n}\right\| \\
& \quad+2 \mu_{1}\left\|z_{n}-p\right\|\left\|B_{1} q-B_{1} v_{n}\right\|+\delta_{n}\left\|A_{1} p\right\|\left(\delta_{n}\left\|A_{1} p\right\|+2\left\|z_{n}-p\right\|\right) \\
& \quad+\left(2+\theta_{n}\right) \theta_{n}\left\|y_{n}-p\right\|^{2}+2 \alpha_{n}\left\|p-w_{n}\right\|\left\|f(p)-\mu A_{2} p\right\| \\
& \leq \\
& \quad\left\|x_{n}-x_{n+1}\right\|\left(\left\|x_{n}-p\right\|+\left\|x_{n+1}-p\right\|\right)+2 \mu_{2}\left\|z_{n}-q\right\|\left\|B_{2} p-B_{2} u_{n}\right\| \\
& \quad+2 \mu_{1}\left\|y_{n}-p\right\|\left\|B_{1} q-B_{1} z_{n}\right\|+\delta_{n}\left\|A_{1} p\right\|\left(\delta_{n}\left\|A_{1} p\right\|+2\left\|z_{n}-p\right\|\right) \\
& \quad+\left(2+\theta_{n}\right) \theta_{n}\left\|y_{n}-p\right\|^{2}+2 \alpha_{n}\left\|p-w_{n}\right\|\left\|f(p)-\mu A_{2} p\right\| .
\end{aligned}
$$

Since $\liminf _{n \rightarrow \infty}\left(1-\beta_{n}\right)>0, \lim _{n \rightarrow \infty} \theta_{n}=0, \lim _{n \rightarrow \infty} \alpha_{n}=0$ and $\lim _{n \rightarrow \infty} \delta_{n}=0$, we conclude from (13) and (21) that

$$
\lim _{n \rightarrow \infty}\left\|u_{n}-v_{n}-(p-q)\right\|=0 \quad \text { and } \quad \lim _{n \rightarrow \infty}\left\|v_{n}-z_{n}+(p-q)\right\|=0 .
$$

It follows that

$$
\left\|u_{n}-G u_{n}\right\|=\left\|u_{n}-z_{n}\right\| \leq\left\|u_{n}-v_{n}-(p-q)\right\|+\left\|v_{n}-z_{n}+(p-q)\right\| \rightarrow 0 \quad(n \rightarrow \infty) .
$$

Also, from (4) we have $\left\|u_{n}-p\right\|^{2} \leq\left(1-\gamma_{n}\right)\left\|u_{n}-p\right\|^{2}+\gamma_{n}\left\langle u_{n}-p, x_{n}-p\right\rangle$, which together with Lemma 2, yields $\left\|u_{n}-p\right\|^{2} \leq\left\langle u_{n}-p, x_{n}-p\right\rangle=\frac{1}{2}\left[\left\|x_{n}-p\right\|^{2}+\left\|u_{n}-p\right\|^{2}-\left\|x_{n}-u_{n}\right\|^{2}\right]$. Thus, we get

$$
\left\|u_{n}-p\right\|^{2} \leq\left\|x_{n}-p\right\|^{2}-\left\|x_{n}-u_{n}\right\|^{2},
$$

which together with (17), yields

$$
\begin{aligned}
& \left\|x_{n+1}-p\right\|^{2} \\
& \leq \beta_{n}\left\|x_{n}-p\right\|^{2}+\left(1-\beta_{n}\right)\left[\left(1-\alpha_{n} \tau\right)\left\|u_{n}-p\right\|^{2}+\delta \alpha_{n}\left\|x_{n}-p\right\|^{2}\right] \\
& \quad+\delta_{n}\left\|A_{1} p\right\|\left(\delta_{n}\left\|A_{1} p\right\|+2\left\|z_{n}-p\right\|\right)+\left(2+\theta_{n}\right) \theta_{n}\left\|y_{n}-p\right\|^{2}+2 \alpha_{n}\left\|p-w_{n}\right\|\left\|f(p)-\mu A_{2} p\right\| \\
& \leq \beta_{n}\left\|x_{n}-p\right\|^{2}+\left(1-\beta_{n}\right)\left\{\alpha_{n} \delta\left\|x_{n}-p\right\|^{2}+\left(1-\alpha_{n} \tau\right)\left[\left\|x_{n}-p\right\|^{2}-\left\|x_{n}-u_{n}\right\|^{2}\right]\right\} \\
& \quad+\delta_{n}\left\|A_{1} p\right\|\left(\delta_{n}\left\|A_{1} p\right\|+2\left\|z_{n}-p\right\|\right)+\left(2+\theta_{n}\right) \theta_{n}\left\|y_{n}-p\right\|^{2}+2 \alpha_{n}\left\|p-w_{n}\right\|\left\|f(p)-\mu A_{2} p\right\| \\
& =\left[1-\alpha_{n}(\tau-\delta)\left(1-\beta_{n}\right)\right]\left\|x_{n}-p\right\|^{2}-\left(1-\alpha_{n} \tau\right)\left(1-\beta_{n}\right)\left\|x_{n}-u_{n}\right\|^{2} \\
& \quad+\delta_{n}\left\|A_{1} p\right\|\left(\delta_{n}\left\|A_{1} p\right\|+2\left\|z_{n}-p\right\|\right)+\left(2+\theta_{n}\right) \theta_{n}\left\|y_{n}-p\right\|^{2}+2 \alpha_{n}\left\|p-w_{n}\right\|\left\|f(p)-\mu A_{2} p\right\| .
\end{aligned}
$$


Hence we have

$$
\begin{aligned}
& \left(1-\alpha_{n} \tau\right)\left(1-\beta_{n}\right)\left\|x_{n}-u_{n}\right\|^{2} \\
& \leq\left\|x_{n}-p\right\|^{2}-\left\|x_{n+1}-p\right\|^{2}+\delta_{n}\left\|A_{1} p\right\|\left(\delta_{n}\left\|A_{1} p\right\|+2\left\|z_{n}-p\right\|\right) \\
& \quad+\left(2+\theta_{n}\right) \theta_{n}\left\|y_{n}-p\right\|^{2}+2 \alpha_{n}\left\|\mu A_{2} p-f(p)\right\|\left\|p-w_{n}\right\| \\
& \leq\left\|x_{n}-x_{n+1}\right\|\left(\left\|x_{n}-p\right\|+\left\|x_{n+1}-p\right\|\right)+\delta_{n}\left\|A_{1} p\right\|\left(\delta_{n}\left\|A_{1} p\right\|+2\left\|z_{n}-p\right\|\right) \\
& \quad+\left(2+\theta_{n}\right) \theta_{n}\left\|y_{n}-p\right\|^{2}+2 \alpha_{n}\left\|f(p)-\mu A_{2} p\right\|\left\|p-w_{n}\right\| .
\end{aligned}
$$

Since $\liminf _{n \rightarrow \infty}\left(1-\beta_{n}\right)>0, \lim _{n \rightarrow \infty} \theta_{n}=0, \lim _{n \rightarrow \infty} \alpha_{n}=0$ and $\lim _{n \rightarrow \infty} \delta_{n}=0$, we obtain from (13) that

$$
\lim _{n \rightarrow \infty}\left\|x_{n}-u_{n}\right\|=0 .
$$

Also, observe that $\left\|x_{n}-z_{n}\right\| \leq\left\|x_{n}-u_{n}\right\|+\left\|G u_{n}-u_{n}\right\|,\left\|x_{n}-G x_{n}\right\| \leq\left\|x_{n}-z_{n}\right\|+\left\|u_{n}-x_{n}\right\|$, and

$$
\left\|x_{n}-y_{n}\right\| \leq\left\|x_{n}-\left(z_{n}-\delta_{n} A_{1} z_{n}\right)\right\| \leq\left\|x_{n}-z_{n}\right\|+\delta_{n}\left\|A_{1} z_{n}\right\| .
$$

Then from (26) and (27) it follows that

$$
\lim _{n \rightarrow \infty}\left\|x_{n}-z_{n}\right\|=0, \quad \lim _{n \rightarrow \infty}\left\|x_{n}-G x_{n}\right\|=0 \quad \text { and } \quad \lim _{n \rightarrow \infty}\left\|x_{n}-y_{n}\right\|=0 .
$$

Step 4. We claim that $\lim _{n \rightarrow \infty}\left\|T x_{n}-x_{n}\right\|=0$ and $\lim _{n \rightarrow \infty}\left\|W_{n} x_{n}-x_{n}\right\|=0$. Indeed, combining (4) and (27), we obtain

$$
\left\|W_{n} u_{n}-u_{n}\right\|=\frac{\gamma_{n}}{1-\gamma_{n}}\left\|x_{n}-u_{n}\right\| \leq \frac{b_{0}}{1-b_{0}}\left\|x_{n}-u_{n}\right\| \rightarrow 0 \quad(n \rightarrow \infty) .
$$

Since each $W_{n}$ is nonexpansive on $C$, from (27) and (29) we get

$$
\begin{aligned}
\left\|W_{n} x_{n}-x_{n}\right\| & \leq\left\|W_{n} u_{n}-u_{n}\right\|+\left\|u_{n}-x_{n}\right\|+\left\|W_{n} x_{n}-W_{n} u_{n}\right\| \\
& \leq\left\|W_{n} u_{n}-u_{n}\right\|+2\left\|u_{n}-x_{n}\right\| \rightarrow 0 \quad(n \rightarrow \infty) .
\end{aligned}
$$

We note that $\left\{\beta_{n}\right\} \subset[c, d]$ and $[c, d] \subset(0,1)$ for some $c, d \in(0,1)$, and observe that

$$
\begin{aligned}
& \left\|x_{n}-T^{n} y_{n}\right\| \leq\left\|x_{n}-x_{n+1}\right\|+\left\|T^{n} y_{n}-x_{n+1}\right\| \\
& \leq\left\|x_{n}-x_{n+1}\right\|+\beta_{n}\left\|x_{n}-T^{n} y_{n}\right\|+\left(1-\beta_{n}\right)\left\|T^{n} y_{n}-P_{C}\left[\left(I-\alpha_{n} \mu A_{2}\right) T^{n} y_{n}+\alpha_{n} f\left(x_{n}\right)\right]\right\| \\
& \leq\left\|x_{n}-x_{n+1}\right\|+\beta_{n}\left\|x_{n}-T^{n} y_{n}\right\|+\left(1-\beta_{n}\right) \alpha_{n}\left(\left\|\mu A_{2}\left(T^{n} y_{n}\right)\right\|+\left\|f\left(x_{n}\right)\right\|\right) .
\end{aligned}
$$

Then we have

$$
(1-d)\left\|x_{n}-T^{n} y_{n}\right\| \leq\left\|x_{n}-x_{n+1}\right\|+(1-d) \alpha_{n}\left(\left\|f\left(x_{n}\right)\right\|+\left\|\mu A_{2}\left(T^{n} y_{n}\right)\right\|\right) .
$$

Hence we get

$$
\begin{aligned}
(1-d)\left\|y_{n}-T^{n} y_{n}\right\| & \leq(1-d)\left\|y_{n}-x_{n}\right\|+(1-d)\left\|x_{n}-T^{n} y_{n}\right\| \\
& \leq(1-d)\left\|y_{n}-x_{n}\right\|+\left\|x_{n}-x_{n+1}\right\|+\alpha_{n}(1-d)\left(\left\|f\left(x_{n}\right)\right\|+\left\|\mu A_{2}\left(T^{n} y_{n}\right)\right\|\right) .
\end{aligned}
$$

Consequently, from (13), (28) and $\lim _{n \rightarrow \infty} \alpha_{n}=0$, it follows that

$$
\lim _{n \rightarrow \infty}\left\|x_{n}-T^{n} y_{n}\right\|=0 \quad \text { and } \quad \lim _{n \rightarrow \infty}\left\|y_{n}-T^{n} y_{n}\right\|=0 .
$$


We also note that

$$
\begin{aligned}
\left\|y_{n}-T y_{n}\right\| & \leq\left\|y_{n}-T^{n} y_{n}\right\|+\left\|T^{n} y_{n}-T^{n+1} y_{n}\right\|+\left\|T^{n+1} y_{n}-T y_{n}\right\| \\
& \leq\left(2+\theta_{1}\right)\left\|T^{n} y_{n}-y_{n}\right\|+\left\|T^{n+1} y_{n}-T^{n} y_{n}\right\| .
\end{aligned}
$$

From $\lim _{n \rightarrow \infty}\left\|T^{n} y_{n}-T^{n+1} y_{n}\right\|=0$ and (31), we get

$$
\lim _{n \rightarrow \infty}\left\|y_{n}-T y_{n}\right\|=0 \text {. }
$$

In addition, noticing that

$$
\left\|x_{n}-T x_{n}\right\| \leq\left\|x_{n}-y_{n}\right\|+\left\|y_{n}-T y_{n}\right\|+\left\|T y_{n}-T x_{n}\right\| \leq\left\|y_{n}-T y_{n}\right\|+\left(2+\theta_{1}\right)\left\|x_{n}-y_{n}\right\|,
$$

we deduce from (28) and (32) that

$$
\lim _{n \rightarrow \infty}\left\|x_{n}-T x_{n}\right\|=0 .
$$

Step 5. We claim that $W: C \rightarrow C$ is nonexpansive, $\operatorname{Fix}(W)=\bigcap_{n=0}^{\infty} \operatorname{Fix}\left(S_{n}\right)$ and $\lim _{n \rightarrow \infty} \| W x_{n}-$ $x_{n} \|=0$ where $W x:=\lim _{n \rightarrow \infty} W_{n} x$ for all $x \in C$. Indeed, we observe that for all $x, y \in C$, $\lim _{n \rightarrow \infty}\left\|W_{n} x-W x\right\|=0$ and $\lim _{n \rightarrow \infty}\left\|W_{n} y-W y\right\|=0$. Since each $W_{n}$ enjoys the nonexpansivity, we get

$$
\|W x-W y\|=\lim _{n \rightarrow \infty}\left\|W_{n} x-W_{n} y\right\| \leq\|x-y\| .
$$

This means that $W$ is nonexpansive. Also, noticing the boundedness of $\left\{x_{n}\right\}$ and putting $D:=$ $\left\{x_{n}: n \geq 0\right\}$, we obtain from Lemma 11 that $\lim _{n \rightarrow \infty} \sup _{x \in D}\left\|W_{n} x-W x\right\|=0$, which immediately sends to

$$
\lim _{n \rightarrow \infty}\left\|W_{n} x_{n}-W x_{n}\right\|=0 .
$$

Thus, combining (30) with (34) we have

$$
\left\|x_{n}-W x_{n}\right\| \leq\left\|x_{n}-W_{n} x_{n}\right\|+\left\|W_{n} x_{n}-W x_{n}\right\| \rightarrow 0 \quad(n \rightarrow \infty) .
$$

In addition, utilizing Lemma 10 we get

$$
\operatorname{Fix}(W)=\bigcap_{n=0}^{\infty} \operatorname{Fix}\left(S_{n}\right)
$$

Step 6. We prove that

$$
\limsup _{n \rightarrow \infty}\left\langle A_{2} x^{*}, x^{*}-w_{n}\right\rangle \leq 0 \text { and } \limsup _{n \rightarrow \infty}\left\langle A_{1} x^{*}, x^{*}-z_{n}\right\rangle \leq 0,
$$

where $\left\{x^{*}\right\}=\operatorname{VI}\left(\operatorname{VI}\left(\Omega, A_{1}\right), \mu A_{2}-f\right)$. Indeed, we choose a subsequence $\left\{w_{n_{i}}\right\}$ of $\left\{w_{n}\right\}$ such that

$$
\limsup _{n \rightarrow \infty}\left\langle x^{*}-w_{n}, A_{2} x^{*}\right\rangle=\lim _{i \rightarrow \infty}\left\langle x^{*}-w_{n_{i}}, A_{2} x^{*}\right\rangle .
$$

Utilizing the boundedness of $\left\{w_{n}\right\} \subset C$, we suppose that $w_{n_{i}} \rightarrow \bar{x} \in C$. Since $\lim _{n \rightarrow \infty} \| x_{n}-$ $T^{n} y_{n} \|=0$ (due to (31)) and $\lim _{n \rightarrow \infty} \alpha_{n}=0$, it follows that

$$
\begin{aligned}
\left\|x_{n}-w_{n}\right\| & \leq\left\|x_{n}-T^{n} y_{n}\right\|+\left\|T^{n} y_{n}-\alpha_{n} f\left(x_{n}\right)-\left(I-\alpha_{n} \mu A_{2}\right) T^{n} y_{n}\right\| \\
& \leq\left\|T^{n} y_{n} x_{n}\right\|+\alpha_{n}\left(\left\|\mu A_{2}\left(T^{n} y_{n}\right)\right\|+\left\|f\left(x_{n}\right)\right\|\right) \rightarrow 0 \quad(n \rightarrow \infty) .
\end{aligned}
$$

Hence, from $w_{n_{i}} \rightarrow \bar{x}$, we get $x_{n_{i}} \rightarrow \bar{x}$. 
Note that $G$ and $W$ are nonexpansive and $T$ is asymptotical. Since $(I-G) x_{n} \rightarrow 0,(I-T) x_{n} \rightarrow 0$ and $(I-W) x_{n} \rightarrow 0$ (due to (28), (33) and (35)), by Lemma 7 we get $\bar{x} \in \operatorname{Fix}(G)=\operatorname{GSVI}\left(C, B_{1}, B_{2}\right)$, $\bar{x} \in \operatorname{Fix}(T)$ and $\bar{x} \in \operatorname{Fix}(W)=\bigcap_{n=0}^{\infty} \operatorname{Fix}\left(S_{n}\right)$. So,

$$
\bar{x} \in \Omega=\bigcap_{n=0}^{\infty} \operatorname{Fix}\left(S_{n}\right) \cap \operatorname{GSVI}\left(C, B_{1}, B_{2}\right) \cap \operatorname{Fix}(T) .
$$

We show $\bar{x} \in \operatorname{VI}\left(\Omega, A_{1}\right)$. Actually, let $y \in \Omega$ be fixed arbitrarily. From (4), (6) and $\zeta$-inverse strong monotonicity of $A_{1}$, we get

$$
\left\|y_{n}-y\right\|^{2} \leq\left\|\left(z_{n}-y\right)-\delta_{n} A_{1} z_{n}\right\|^{2} \leq\left\|x_{n}-y\right\|^{2}+2 \delta_{n}\left\langle y-z_{n}, A_{1} y\right\rangle+\delta_{n}^{2}\left\|A_{1} z_{n}\right\|^{2},
$$

which implies that, for all $n \geq 0$,

$$
\begin{aligned}
0 & \leq \frac{1}{\delta_{n}}\left(\left\|x_{n}-y\right\|^{2}-\left\|y_{n}-y\right\|^{2}\right)+2\left\langle A_{1} y, y-z_{n}\right\rangle+\delta_{n}\left\|A_{1} z_{n}\right\|^{2} \\
& \leq\left(\left\|x_{n}-y\right\|+\left\|y_{n}-y\right\|\right) \frac{\left\|x_{n}-y_{n}\right\|}{\delta_{n}}+2\left\langle A_{1} y, y-z_{n}\right\rangle+\delta_{n}\left\|A_{1} z_{n}\right\|^{2} .
\end{aligned}
$$

From (28) it is easy to see $x_{n_{i}} \rightarrow \bar{x}$ leads to $z_{n_{i}} \rightarrow \bar{x}$. Since $\lim _{n \rightarrow \infty} \delta_{n}=0$ and $\left\|x_{n}-y_{n}\right\|=o\left(\delta_{n}\right)$, we have

$$
\begin{aligned}
0 & \leq \liminf _{n \rightarrow \infty}\left\{\left(\left\|x_{n}-y\right\|+\left\|y_{n}-y\right\|\right) \frac{\left\|x_{n}-y_{n}\right\|}{\delta_{n}}+2\left\langle A_{1} y, y-z_{n}\right\rangle+\delta_{n}\left\|A_{1} z_{n}\right\|^{2}\right\} \\
& =\liminf _{n \rightarrow \infty}\left\langle y-z_{n}, A_{1} y\right\rangle \leq \lim _{i \rightarrow \infty} 2\left\langle y-z_{n_{i}}, A_{1} y\right\rangle=2\left\langle y-\bar{x}, A_{1} y\right\rangle .
\end{aligned}
$$

It follows that $\left\langle A_{1} y, y-\bar{x}\right\rangle \geq 0, \forall y \in \Omega$. So, Lemma 12 and the $\zeta$-inverse-strong monotonicity of $A_{1}$ ensure that $\left\langle y-\bar{x}, A_{1} \bar{x}\right\rangle \geq 0, \quad \forall y \in \Omega$, that is, $\bar{x} \in \operatorname{VI}\left(\Omega, A_{1}\right)$. Consequently, from $\left\{x^{*}\right\}=$ $\operatorname{VI}\left(\operatorname{VI}\left(\Omega, A_{1}\right), \mu A_{2}-f\right)$, we have

$$
\limsup _{n \rightarrow \infty}\left\langle x^{*}-w_{n},\left(\mu A_{2}-f\right) x^{*}\right\rangle=\lim _{i \rightarrow \infty}\left\langle x^{*}-w_{n_{i}},\left(\mu A_{2}-f\right) x^{*}\right\rangle=\left\langle x^{*}-\bar{x},\left(\mu A_{2}-f\right) x^{*}\right\rangle \leq 0 .
$$

Also, we pick a subsequence $\left\{z_{n_{k}}\right\} \subset\left\{z_{n}\right\}$ such that

$$
\limsup _{n \rightarrow \infty}\left\langle x^{*}-z_{n}, A_{1} x^{*}\right\rangle=\lim _{k \rightarrow \infty}\left\langle x^{*}-z_{n_{k}}, A_{1} x^{*}\right\rangle .
$$

Since vector sequence $\left\{z_{n}\right\}$ is bounded in $C$, we suppose that $z_{n_{k}} \rightarrow \hat{x} \in C$. From (28) it is clear that $z_{n_{k}} \rightarrow \hat{x}$ yields $x_{n_{k}} \rightarrow \hat{x}$. By the same arguments as in the proof of $\bar{x} \in \Omega$, we have $\hat{x} \in \Omega$. From $x^{*} \in \operatorname{VI}\left(\Omega, A_{1}\right)$, we get

$$
\limsup _{n \rightarrow \infty}\left\langle x^{*}-z_{n}, A_{1} x^{*}\right\rangle=\lim _{k \rightarrow \infty}\left\langle x^{*}-z_{n_{k}}, A_{1} x^{*}\right\rangle=\left\langle x^{*}-\hat{x}, A_{1} x^{*}\right\rangle \leq 0 .
$$

Therefore, the inequalities in (37) hold.

Step 7. We propose $x_{n} \rightarrow x^{*}$ as $n \rightarrow \infty$. Indeed, putting $p=x^{*}$ in (6) and (16) we obtain that $\left\|z_{n}-x^{*}\right\| \leq\left\|x_{n}-x^{*}\right\|$ and

$$
\left\|w_{n}-x^{*}\right\|^{2} \leq \alpha_{n} \delta\left\|x_{n}-x^{*}\right\|^{2}+\left(1-\alpha_{n} \tau\right)\left\|y_{n}-x^{*}\right\|^{2}+\theta_{n}\left(2+\theta_{n}\right)\left\|y_{n}-x^{*}\right\|^{2}+2 \alpha_{n}\left\langle\left(\mu A_{2}-f\right) x^{*}, x^{*}-w_{n}\right\rangle .
$$

From (4) and the $\zeta$-inverse-strong monotonicity of $A_{1}$ it follows that

$$
\left\|y_{n}-x^{*}\right\|^{2} \leq\left\|\left(z_{n}-x^{*}\right)-\delta_{n} A_{1} z_{n}\right\|^{2} \leq\left\|x_{n}-x^{*}\right\|^{2}+2 \delta_{n}\left\langle A_{1} x^{*}, x^{*}-z_{n}\right\rangle+\delta_{n}^{2}\left\|A_{1} z_{n}\right\|^{2} .
$$


Thus, in terms of (4), (39) and (40), we get

$$
\begin{aligned}
& \left\|x_{n+1}-x^{*}\right\|^{2} \leq\left(1-\beta_{n}\right)\left\|w_{n}-x^{*}\right\|^{2}+\beta_{n}\left\|x_{n}-x^{*}\right\|^{2} \\
& \leq\left(1-\beta_{n}\right)\left[\alpha_{n} \delta\left\|x_{n}-x^{*}\right\|^{2}+\left(1-\alpha_{n} \tau\right)\left\|y_{n}-x^{*}\right\|^{2}+\theta_{n}\left(2+\theta_{n}\right)\left\|y_{n}-x^{*}\right\|^{2}+\beta_{n}\left\|x_{n}-x^{*}\right\|^{2}\right. \\
& \left.\quad+2 \alpha_{n}\left\langle\left(\mu A_{2}-f\right) x^{*}, x^{*}-w_{n}\right\rangle\right] \\
& \leq \beta_{n}\left\|x_{n}-x^{*}\right\|^{2}+\left(1-\beta_{n}\right)\left\{\alpha_{n} \delta\left\|x_{n}-x^{*}\right\|^{2}+\left(1-\alpha_{n} \tau\right)\left[2 \delta_{n}\left\langle A_{1} x^{*}, x^{*}-z_{n}\right\rangle+\left\|x_{n}-x^{*}\right\|^{2}\right.\right. \\
& \left.\left.\quad+\delta_{n}^{2}\left\|A_{1} z_{n}\right\|^{2}\right]+\theta_{n}\left(2+\theta_{n}\right)\left\|y_{n}-x^{*}\right\|^{2}+2 \alpha_{n}\left\langle\left(\mu A_{2}-f\right) x^{*}, x^{*}-w_{n}\right\rangle\right\} \\
& \leq \\
& \quad\left[1-\alpha_{n}(\tau-\delta)\left(1-\beta_{n}\right)\right]\left\|x_{n}-x^{*}\right\|^{2}+\alpha_{n}(\tau-\delta)\left(1-\beta_{n}\right)\left\{\frac{\left(1-\alpha_{n} \tau\right) 2 \delta_{n}}{(\tau-\delta) \alpha_{n}}\left\langle x^{*}-z_{n}, A_{1} x^{*}\right\rangle\right. \\
& \left.\quad+\alpha_{n} \frac{\left\|A_{1} z_{n}\right\|^{2}}{\tau-\delta}+\frac{\left(2+\theta_{n}\right) \theta_{n}\left\|y_{n}-x^{*}\right\|^{2}}{(\tau-\delta) \alpha_{n}}+\frac{2}{\tau-\delta}\left\langle\left(\mu A_{2}-f\right) x^{*}, x^{*}-w_{n}\right\rangle\right\} .
\end{aligned}
$$

Obviously, (37) yields

$$
\limsup _{n \rightarrow \infty} \frac{\left(1-\alpha_{n} \tau\right) 2 \delta_{n}}{(\tau-\delta) \alpha_{n}}\left\langle x^{*}-z_{n}, A_{1} x^{*}\right\rangle \leq 0
$$

and

$$
\limsup _{n \rightarrow \infty} \frac{2}{\tau-\delta} \cdot\left\langle x^{*}-w_{n},\left(\mu A_{2}-f\right) x^{*}\right\rangle \leq 0 .
$$

Actually, from $\lim \sup _{n \rightarrow \infty}\left\langle A_{1} x^{*}, x^{*}-z_{n}\right\rangle \leq 0$ it follows that for any given $\varepsilon>0$ there exists an integer $n_{0} \geq 1$ such that $\left\langle A_{1} x^{*}, x^{*}-z_{n}\right\rangle \leq \varepsilon, \forall n \geq n_{0}$. Then from $\delta_{n} \leq \alpha_{n}$ we get

$$
\frac{2 \delta_{n}\left(1-\alpha_{n} \tau\right)}{(\tau-\delta) \alpha_{n}}\left\langle A_{1} x^{*}, x^{*}-z_{n}\right\rangle \leq \frac{2 \delta_{n}\left(1-\alpha_{n} \tau\right)}{(\tau-\delta) \alpha_{n}} \varepsilon \leq \frac{2}{\tau-\delta} \varepsilon, \quad \forall n \geq n_{0},
$$

which hence yields

$$
\limsup _{n \rightarrow \infty} \frac{2 \delta_{n}\left(1-\alpha_{n} \tau\right)\left\langle A_{1} x^{*}, x^{*}-z_{n}\right\rangle}{(\tau-\delta) \alpha_{n}} \leq \frac{2}{\tau-\delta} \varepsilon .
$$

Letting $\varepsilon \rightarrow 0$, we get

$$
\limsup _{n \rightarrow \infty} \frac{2 \delta_{n}\left(1-\alpha_{n} \tau\right)\left\langle x^{*}-z_{n}, A_{1} x^{*}\right\rangle}{(\tau-\delta) \alpha_{n}} \leq 0 .
$$

Since $\sum_{n=0}^{\infty} \alpha_{n}=\infty, \liminf _{n \rightarrow \infty}\left(1-\beta_{n}\right)>0$ and $\lim _{n \rightarrow \infty} \frac{\theta_{n}}{\alpha_{n}}=0$, we deduce that

$$
\sum_{n=0}^{\infty} \alpha_{n}(\tau-\delta)\left(1-\beta_{n}\right)=\infty
$$

and

$$
\begin{aligned}
\limsup _{n \rightarrow \infty} & \left\{\frac{\left(1-\alpha_{n} \tau\right) 2 \delta_{n}}{(\tau-\delta) \alpha_{n}}\left\langle A_{1} x^{*}, x^{*}-z_{n}\right\rangle+\alpha_{n} \frac{\left\|A_{1} z_{n}\right\|^{2}}{\tau-\delta}\right. \\
& \left.+\frac{\theta_{n}\left(2+\theta_{n}\right)\left\|y_{n}-x^{*}\right\|^{2}}{(\tau-\delta) \alpha_{n}}+\frac{2}{\tau-\delta}\left\langle x^{*}-w_{n},\left(\mu A_{2}-f\right) x^{*}\right\rangle\right\} \leq 0 .
\end{aligned}
$$

We can infer Lemma 3 to the relation (41) and conclude that $x_{n} \rightarrow x^{*}$ as $n \rightarrow \infty$. This completes the proof.

From Theorem 1, we have the following sub-result.

Corollary 1. Assume that $\mu_{1}$ is a real number in $(0,2 \alpha)$, and $\mu_{2}$ is a real number in $(0,2 \beta)$. Let $\delta<\tau:=$ $1-\sqrt{1-\mu\left(2 \eta-\mu \kappa^{2}\right)} \in(0,1]$ for $\mu \in\left(0, \frac{2 \eta}{\kappa^{2}}\right)$. We suppose $\left\{\lambda_{n}\right\}_{n=0}^{\infty}$ is a real sequence in $(0, b]$ for some real number $b$ in $(0,1)$. We also suppose that $\left\{\alpha_{n}\right\},\left\{\beta_{n}\right\},\left\{\gamma_{n}\right\} \subset(0,1]$ and $\left\{\delta_{n}\right\} \subset(0,2 \zeta]$ such that

(i) $\sum_{n=0}^{\infty} \alpha_{n}=\infty$ and $\lim _{n \rightarrow \infty} \alpha_{n}=0$;

(ii) $\delta_{n} \leq \alpha_{n} \forall n \geq 0$ and $\lim _{n \rightarrow \infty} \frac{\theta_{n}}{\alpha_{n}}=0$;

(iii) $\liminf _{n \rightarrow \infty} \beta_{n}>0$ and $\lim \sup _{n \rightarrow \infty} \beta_{n}<1$; 
(iv) $\liminf _{n \rightarrow \infty} \gamma_{n}>0$, limsup $\sup _{n \rightarrow \infty} \gamma_{n}<1$ and $\lim _{n \rightarrow \infty}\left|\gamma_{n+1}-\gamma_{n}\right|=0$;

(v) $\lim _{n \rightarrow \infty}\left\|T^{n+1} y_{n}-T^{n} y_{n}\right\|=0$.

Let $\left\{x_{n}\right\}_{n=0}^{\infty}$ be a sequence defined by

$$
\left\{\begin{array}{l}
y_{n}=\left(1-\gamma_{n}\right) W_{n} y_{n}+\gamma_{n} x_{n} \\
x_{n+1}=\beta_{n} x_{n}+\left(1-\beta_{n}\right) P_{C}\left[\alpha_{n} f\left(x_{n}\right)+\left(I-\alpha_{n} \mu A_{2}\right) T^{n} y_{n}\right]
\end{array}\right.
$$

Then we have

(a) $\left\{x_{n}\right\}_{n=0}^{\infty}$ is bounded;

(b) $\lim _{n \rightarrow \infty}\left\|x_{n}-y_{n}\right\|=0, \lim _{n \rightarrow \infty}\left\|x_{n}-T x_{n}\right\|=0$ and $\lim _{n \rightarrow \infty}\left\|x_{n}-W x_{n}\right\|=0$;

(c) if $\lim _{n \rightarrow} \frac{\left\|x_{n}-y_{n}\right\|}{\delta_{n}}=0$, then $\left\{x_{n}\right\}$ converges to a common fixed point of the asymptotically nonexpansive and nonexpansive mappings.

Theorem 2. Assume that $\mu_{1}$ is a real number in $(0,2 \alpha)$, and $\mu_{2}$ is a real number in $(0,2 \beta)$. Let $\tau=$ $1-\sqrt{1-\mu\left(2 \eta-\mu \kappa^{2}\right)} \in(0,1]$ for $\mu$ in $\left(0, \frac{2 \eta}{\kappa^{2}}\right)$, and let $\left\{\lambda_{n}\right\}_{n=0}^{\infty}$ be a real sequence in $(0, b]$ for some $b$ in $(0,1)$. Suppose that $\left\{\alpha_{n}\right\},\left\{\beta_{n}\right\},\left\{\gamma_{n}\right\} \subset(0,1]$ and $\left\{\delta_{n}\right\} \subset(0,2 \zeta]$ such that

(i) $\sum_{n=0}^{\infty} \alpha_{n}=\infty$ and $\lim _{n \rightarrow \infty} \alpha_{n}=0$;

(ii) $\delta_{n} \leq \alpha_{n} \forall n \geq 0$ and $\lim _{n \rightarrow \infty} \frac{\theta_{n}}{\alpha_{n}}=0$;

(iii) $\liminf _{n \rightarrow \infty} \beta_{n}>0$ and $\limsup _{n \rightarrow \infty} \beta_{n}<1$;

(iv) $\liminf _{n \rightarrow \infty} \gamma_{n}>0$, limsup $\sup _{n \rightarrow \infty} \gamma_{n}<1$ and $\lim _{n \rightarrow \infty}\left|\gamma_{n+1}-\gamma_{n}\right|=0$;

(v) $\lim _{n \rightarrow \infty}\left\|T^{n+1} y_{n}-T^{n} y_{n}\right\|=0$.

Then the sequence $\left\{x_{n}\right\}_{n=0}^{\infty}$ generated by Algorithm 3 satisfies the following properties:
(a) $\left\{x_{n}\right\}_{n=0}^{\infty}$ is bounded;
(b) $\lim _{n \rightarrow \infty}\left\|x_{n}-y_{n}\right\|=0, \lim _{n \rightarrow \infty}\left\|x_{n}-G x_{n}\right\|=0, \lim _{n \rightarrow \infty}\left\|x_{n}-T x_{n}\right\|=0$ and $\lim _{n \rightarrow \infty} \| x_{n}-$ $W x_{n} \|=0$;
(c) If $\frac{\left\|x_{n}-y_{n}\right\|}{\delta_{n}}=0, x_{n} \rightarrow x^{*} \in \mathrm{VI}\left(\Omega, A_{1}\right)$.

Proof. Since $A_{2}: C \rightarrow H$ is $\kappa$-Lipschitzian and $\eta$-strongly monotone, by Lemma 12 we know that the Problem 2 has the unique solution. We let $\left\{x^{*}\right\}=\operatorname{VI}\left(\operatorname{VI}\left(\Omega, A_{1}\right), A_{2}\right)$. For each $n \geq 0$, we consider the mapping $F_{n} x:=G\left(\gamma_{n} x_{n}+\left(1-\gamma_{n}\right) W_{n} x\right), \forall x \in C$. Utilizing the same argument as in the proof of Theorem 1, we can deduce from Banach's contraction principle that for each $n \geq 0$ there exists a unique element $z_{n} \in C$ such that $z_{n}=G\left(\gamma_{n} x_{n}+\left(1-\gamma_{n}\right) W_{n} z_{n}\right)$. Thus, the iterative scheme in Algorithm 3 can be rewritten as

$$
\left\{\begin{array}{l}
u_{n}=\gamma_{n} x_{n}+\left(1-\gamma_{n}\right) W_{n} z_{n}, \\
z_{n}=G u_{n} \\
y_{n}=P_{C}\left(z_{n}-\delta_{n} A_{1} z_{n}\right), \\
x_{n+1}=\beta_{n} x_{n}+\left(1-\beta_{n}\right) P_{C}\left(I-\mu \alpha_{n} A_{2}\right) T^{n} y_{n} .
\end{array}\right.
$$

Here, we divide the rest of the proof into several steps.

Step 1. We prove $\left\{x_{n}\right\},\left\{y_{n}\right\},\left\{z_{n}\right\},\left\{u_{n}\right\},\left\{v_{n}\right\},\left\{T^{n} y_{n}\right\}$ and $\left\{A_{2}\left(T^{n} y_{n}\right)\right\}$ are bounded vector sequences, where $v_{n}=P_{C}\left(u_{n}-\mu_{2} B_{2} u_{n}\right)$ and $z_{n}=P_{C}\left(v_{n}-\mu_{1} B_{1} v_{n}\right)$ for all $n \geq 0$. Indeed, utilizing the similar argument to that of Step 1 in the proof of Theorem 1, we obtain the desired assertion.

Step 2. We prove $\left\|x_{n+1}-x_{n}\right\| \rightarrow 0$ and $\left\|y_{n+1}-y_{n}\right\| \rightarrow 0$ as $n \rightarrow \infty$. Indeed, utilizing the similar argument to that of Step 2 in the proof of Theorem 1, we obtain the desired assertion.

Step 3. We prove $\left\|x_{n}-G x_{n}\right\| \rightarrow 0$ as $n \rightarrow \infty$. Indeed, utilizing the similar argument to that of Step 3 in the proof of Theorem 1, we obtain the desired assertion.

Step 4. We prove $\left\|T x_{n}-x_{n}\right\| \rightarrow 0$ and $\left\|W_{n} x_{n}-x_{n}\right\| \rightarrow 0$ as $n \rightarrow \infty$. Indeed, utilizing the similar argument to that of Step 4 in the proof of Theorem 1, we obtain the desired assertion.

Step 5. We prove $W: C \rightarrow C$ enjoys the nonexpansivity, $\operatorname{Fix}(W)=\bigcap_{n=0}^{\infty} \operatorname{Fix}\left(S_{n}\right)$ and $\lim _{n \rightarrow \infty}\left\|W x_{n}-x_{n}\right\|=0$ where $W x:=\lim _{n \rightarrow \infty} W_{n} x$ for all $x \in C$. Indeed, utilizing the similar argument to that of Step 5 in the proof of Theorem 1, we obtain the desired assertion. 
Step 6. We prove $\lim \sup _{n \rightarrow \infty}\left\langle A_{2} x^{*}, x^{*}-w_{n}\right\rangle \leq 0$ and $\limsup _{n \rightarrow \infty}\left\langle A_{1} x^{*}, x^{*}-z_{n}\right\rangle \leq 0$, where $\left\{x^{*}\right\}=\operatorname{VI}\left(\operatorname{VI}\left(\Omega, A_{1}\right), A_{2}\right)$. Indeed, utilizing the similar argument to that of Step 6 in the proof of Theorem 1, we obtain the desired assertion.

Step 7. We prove $x_{n} \rightarrow x^{*}$ as $n \rightarrow \infty$. Indeed, utilizing the similar argument to that of Step 7 in the proof of Theorem 1, we obtain the desired assertion.

This completes the entire proof.

Corollary 2. Assume that $\mu_{1}$ is a real number in $(0,2 \alpha)$, and $\mu_{2}$ is a real number in $(0,2 \beta)$. Let $\tau=$ $1-\sqrt{1-\mu\left(2 \eta-\mu \kappa^{2}\right)} \in(0,1]$ for $\mu$ in $\left(0, \frac{2 \eta}{\kappa^{2}}\right)$, and let $\left\{\lambda_{n}\right\}_{n=0}^{\infty}$ be a real sequence in $(0, b]$ for some $b$ in $(0,1)$. Suppose that $\left\{\alpha_{n}\right\},\left\{\beta_{n}\right\},\left\{\gamma_{n}\right\} \subset(0,1]$ and $\left\{\delta_{n}\right\} \subset(0,2 \zeta]$ such that

(i) $\sum_{n=0}^{\infty} \alpha_{n}=\infty$ and $\lim _{n \rightarrow \infty} \alpha_{n}=0$;

(ii) $\delta_{n} \leq \alpha_{n} \forall n \geq 0$ and $\lim _{n \rightarrow \infty} \frac{\theta_{n}}{\alpha_{n}}=0$;

(iii) $\liminf _{n \rightarrow \infty} \beta_{n}>0$ and $\lim \sup _{n \rightarrow \infty} \beta_{n}<1$;

(iv) $\liminf _{n \rightarrow \infty} \gamma_{n}>0$, limsup $\sup _{n \rightarrow \infty} \gamma_{n}<1$ and $\lim _{n \rightarrow \infty}\left|\gamma_{n+1}-\gamma_{n}\right|=0$;

(v) $\lim _{n \rightarrow \infty}\left\|T^{n+1} y_{n}-T^{n} y_{n}\right\|=0$.

Let $\left\{x_{n}\right\}_{n=0}^{\infty}$ be a sequence defined by

$$
\left\{\begin{array}{l}
u_{n}=\left(1-\gamma_{n}\right) W_{n} u_{n}+\gamma_{n} x_{n} \\
x_{n+1}=\beta_{n} x_{n}+\left(1-\beta_{n}\right) P_{C}\left(I-\alpha_{n} \mu A_{2}\right) T^{n} u_{n}
\end{array}\right.
$$

Then we have

(a) $\left\{x_{n}\right\}_{n=0}^{\infty}$ is bounded;

(b) $\lim _{n \rightarrow \infty}\left\|x_{n}-u_{n}\right\|=0, \lim _{n \rightarrow \infty}\left\|x_{n}-T x_{n}\right\|=0$ and $\lim _{n \rightarrow \infty}\left\|x_{n}-W x_{n}\right\|=0$;

(c) If $\frac{\left\|x_{n}-u_{n}\right\|}{\delta_{n}}=0,\left\{x_{n}\right\}$

converges to a common fixed point of the asymptotically nonexpansive and nonexpansive mappings.

\section{Concluding Remark}

This paper discussed a monotone variational inequality problem with a variational inequality constraint over the common solution set of a general system of variational inequalities and a common fixed point of a countable family of nonexpansive mappings and an asymptotically nonexpansive mapping in Hilbert spaces, which is called the triple hierarchical constrained variational inequality, and introduced some Mann-type implicit iteration methods for solving it. Norm convergence of the proposed methods of the iteration methods is guaranteed under some suitable assumptions.

Author Contributions: These authors contributed equally to this work.

Funding: This research was partially funded by supported by the Innovation Program of Shanghai Municipal Education Commission (15ZZ068), Ph.D. Program Foundation of Ministry of Education of China (20123127110002) and Program for Outstanding Academic Leaders in Shanghai City (15XD1503100).

Acknowledgments: The authors are grateful to the editor and the referees for useful suggestions which improved the contents of this paper.

Conflicts of Interest: The authors declare no conflict of interest.

\section{References}

1. Goebel, K.; Kirk, W.A. A fixed point theorem for asymptotically nonexpansive mappings. Proc. Am. Math. Soc. 1972, 35, 171-174. [CrossRef]

2. Qin, X.; Petrusel, A.; Yao, J.C. CQ iterative algorithms for fixed points of nonexpansive mappings and split feasibility problems in Hilbert spaces. J. Nonlinear Convex Anal. 2018, 19, 157-165

3. Chang, S.S.; Wen, C.F.; Yao, J.C. Zero point problem of accretive operators in Banach spaces. Bull. Malays. Math. Sci. Soc. 2019, 42, 105-118. [CrossRef] 
4. Cho, S.Y.; Qin, X. On the strong convergence of an iterative process for asymptotically strict pseudocontractions and equilibrium problems. Appl. Math. Comput. 2014, 235, 430-438. [CrossRef]

5. Cho, S.Y. Strong convergence analysis of a hybrid algorithm for nonlinear operators in a Banach space. J. Appl. Anal. Comput. 2016, 9, 1083-1092.

6. Ceng, L.C. Approximation of common solutions of a split inclusion problem and a fixed-point problem. J. Appl. Numer. Optim. 2019, 1, 1-12.

7. Alsulami, S.M.; Latif, A.; Takahashi, W. The split common fixed point problem and strong convergence theorems by hybrid methods for new demimetric mappings in Hilbert spaces. Appl. Anal. Optim. 2018, 2, 11-26.

8. Ceng, L.C. Petrusel, A. Hybrid viscosity extragradient method for systems of variational inequalities, fixed points of nonexpansive mappings, zero points of accretive operators in Banach spaces. Fixed Point Theory 2018, 19, 487-502. [CrossRef]

9. Ceng, L.C.; Al-Otaibi, A.; Ansari, Q.H.; Latif, A. Relaxed and composite viscosity methods for variational inequalities, fixed points of nonexpansive mappings and zeros of accretive operators. Fixed Point Theory Appl. 2014, 2014, 29. [CrossRef]

10. Qin, X.; Cho, S.Y.; Wang, L. Strong convergence of an iterative algorithm involving nonlinear mappings of nonexpansive and accretive type. Optimization 2018 67, 1377-1388. [CrossRef]

11. Zhao, X.; Ng, K.F.; Li, C.; Yao, J.C. Linear regularity and linear convergence of projection-based methods for solving convex feasibility problems. Appl. Math. Optim. 2018, 78, 613-641. [CrossRef]

12. Cho, S.Y.; Dehaish, B.A.B.; Qin, X. Weak convergence of a splitting algorithm in Hilbert spaces. J. Appl. Anal. Comput. 2017, 7, 427-438.

13. Cho, S.Y. Viscosity approximation splitting methods for monotone and nonexpansive operators in Hilbert spaces. J. Nonlinear Convex Anal. 2018, 19, 251-264.

14. Yao, Y.; Qin, X.; Yao, J.C. Projection methods for firmly type nonexpansive operators. J. Nonlinear Convex Anal. 2018, 19, 407-415.

15. Dehaish, B.A.B. Weak and strong convergence of algorithms for the sum of two accretive operators with applications. J. Nonlinear Convex Anal. 2015, 16, 1321-1336.

16. Chang, S.S.; Wen, C.F.; Yao, J.C. Common zero point for a finite family of inclusion problems of accretive mappings in Banach spaces. Optimization 2018, 67, 1183-1196. [CrossRef]

17. Ceng, L.C.; Yuan, Q. Hybrid Mann viscosity implicit iteration methods for triple hierarchical variational inequalities, systems of variational inequalities and fixed point problems. Mathematics 2019, 7, 142. [CrossRef]

18. Nguyen, L.V. Some results on strongly pseudomonotone quasi-variational inequalities. Set-Valued Var. Anal. 2019. [CrossRef]

19. Ceng, L.C.; Wang, C.Y.; Yao, J.C. Strong convergence theorems by a relaxed extragradient method for a general system of variational inequalities. Math. Methods Oper. Res. 2008, 67, 375-390. [CrossRef]

20. Cai, G.; Shehu, Y.; Iyiola, O.S. Strong convergence results for variational inequalities and fixed point problems using modified viscosity implicit rules. Numer. Algorithms 2018, 77, 535-558. [CrossRef]

21. Iiduka, H. Iterative algorithm for solving triple-hierarchical constrained optimization problem. J. Optim. Theory Appl. 2011, 148, 580-592. [CrossRef]

22. Yao, Y.; Postolache, H.; Liou, Y.C. Variant extragradient-type method for monotone variational inequalities. Fixed Point Theory Appl. 2013, 2013, 185. [CrossRef]

23. Yao, Y.; Postolache, H. Iterative methods for pseudomonotone variational inequalities and fixed-point problems. J. Optim. Theory Appl. 2012, 155, 273-287. [CrossRef]

24. Qin, X.; Yao, J.C. Projection splitting algorithms for nonself operators. J. Nonlinear Convex Anal. 2017, 18, 925-935.

25. Yao, Y.; Yao, J.C.; Liou, Y.C.; Postolache, M. Iterative algorithms for split common fixed points of demicontractive operators without priori knowledge of operator norms. Carpathian J. Math. 2018, 34, 459-466.

26. Qin, X.; Cho, S.Y. Convergence analysis of a monotone projection algorithm in reflexive Banach spaces. Acta Math. Sci. 2017, 37, 488-502. [CrossRef]

27. Xu, H.K.; Kim, T.H. Convergence of hybrid steepest-descent methods for variational inequalities. J. Optim. Theory Appl. 2003, 119, 185-201. [CrossRef]

28. Ceng, L.C.; Xu, H.K.; Yao, J.C. The viscosity approximation method for asymptotically nonexpansive mappings in Banach spaces. Nonlinear Anal. 2008, 69, 1402-1412. [CrossRef] 
29. Suzuki, T. Strong convergence of Krasnoselskii and Mann's type sequences for one-parameter nonexpansive semigroups without Bochner integrals. J. Math. Anal. Appl. 2005, 305, 227-239. [CrossRef]

30. Shimoji, K.; Takahashi, W. Strong convergence to common fixed points of infinite nonexpansive mappings and applications. Taiwan. J. Math. 2001, 5, 387-404. [CrossRef]

(C) 2019 by the authors. Licensee MDPI, Basel, Switzerland. This article is an open access article distributed under the terms and conditions of the Creative Commons Attribution (CC BY) license (http:/ / creativecommons.org/licenses/by/4.0/). 